\title{
Molecular Design, Optimization, and Genomic Integration of Chimeric B Cell Receptors in Murine B Cells
}

\author{
Theresa Pesch ${ }^{1}$, Lucia Bonati ${ }^{1}$, William Kelton ${ }^{1}$, Cristina Parola ${ }^{1,2}$, Roy A. Ehling ${ }^{1}$, \\ Lucia Csepregi ${ }^{1,3}$, Daisuke Kitamura ${ }^{4}$ and Sai T. Reddy ${ }^{1 *}$
}

${ }^{1}$ Department of Biosystems Science and Engineering, ETH Zürich, Basel, Switzerland, ${ }^{2}$ Life Science Graduate School, Systems Biology, ETH Zürich, University of Zurich, Zurich, Switzerland, ${ }^{3}$ Life Science Graduate School, Microbiology and Immunology, ETH Zürich, University of Zurich, Zurich, Switzerland, ${ }^{4}$ Research Institute for Biomedical Sciences, Tokyo University of Science, Noda, Japan

Immune cell therapies based on the integration of synthetic antigen receptors comprise a powerful strategy for the treatment of diverse diseases, most notably $T$ cells engineered to express chimeric antigen receptors (CAR) for targeted cancer therapy. In addition to $T$ lymphocytes, B lymphocytes may also represent valuable immune cells that can be engineered for therapeutic purposes such as protein replacement therapy or recombinant antibody production. In this article, we report a promising concept for the molecular design, optimization, and genomic integration of a novel class of synthetic antigen receptors, chimeric B cell receptors (CBCR). We initially optimized CBCR expression and detection by modifying the extracellular surface tag, the transmembrane regions and intracellular signaling domains. For this purpose, we stably integrated a series of CBCR variants using CRISPR-Cas9 into immortalized B cell hybridomas. Subsequently, we developed a reliable and consistent pipeline to precisely introduce cassettes of several kb size into the genome of primary murine B cells also using CRISPR-Cas9 induced HDR. Finally, we were able to show the robust surface expression and antigen recognition of a synthetic CBCR in primary B cells. We anticipate CBCRs and our approach for engineering primary B cells will be a valuable tool for the advancement of future B cell- based immune cell therapies.

Keywords: B cells, synthetic antigen receptor, cellular engineering, genome editing, cellular immunotherapy, CRISPR-Cas9

\section{INTRODUCTION}

The successful clinical results of genetically modified $\mathrm{T}$ cells for cancer immunotherapy have shown the great potential for engineering immune cells for cellular medicine (1-4). Engineered $\mathrm{CD} 8^{+} \mathrm{T}$ cells have shown the most progress as they can execute cytotoxic functions by inducing target cells to undergo programmed cell death (5), thus providing a means to directly attack cancer cells. The strategy to take advantage of the natural features of immune cells, while re-directing their specificity by receptor engineering has culminated in the concept of chimeric antigen receptor (CAR) T cells (6-8). A CAR is a recombinant antigen receptor composed of an extracellular antigen-binding domain, typically an antibody fragment [e.g., a single-chain variable fragment (scFv)], linked by a spacer peptide to a transmembrane domain, which is further fused to an intracellular $\mathrm{T}$ cell activation domain, such as $\mathrm{CD} 3 \zeta(9-11)$. A broad range of extracellular binding domains and intracellular costimulatory domains (e.g., CD28 and 4-1BB) have been incorporated into CARs 
to further enhance their targeting and signaling properties (12-16). CAR $\mathrm{T}$ cell therapies rely on the isolation, the ex vivo expansion and engineering of $\mathrm{T}$ lymphocytes by the introduction of CARs followed by the re-introduction into the patient. While the engineering and development of $\mathrm{T}$ cells as cellular therapeutics is advancing rapidly, B lymphocytes represent another class of immune cells that hold promise of being powerful vehicles for adoptive cell therapy due to their involvement in essential processes of immunological recognition and protection. Considering the similarity in the principle of clonal selection and expansion upon antigen exposure, it might be possible to take advantage of natural features of $\mathrm{B}$ cells for therapeutic purposes. For example, B cells have very interesting innate properties, such as their ability to differentiate, following antigen-specific activation, into long-lived antibody secreting plasma cells, which home to and reside in specific bone marrow niches, reportedly for decades $(17,18)$. Their longevity and known requirements to secrete large quantities of protein make primary B cells unique and promising targets as cellular host for therapeutic protein production (19).

Primary T cells can be genetically modified (via lentiviral or retroviral integration) and expanded in vitro relatively easily, in contrast, progress on engineering of $\mathrm{B}$ cells has been severely compromised by technical challenges in their in vitro culture, expansion, and genetic modification. This may be the reason that B cells have received relatively little attention as cellular engineering hosts in immunotherapy. While high rates of transduction in B cells can be obtained using recombinant adenovirus or Epstein-Barr virus vectors, this only results in temporarily expression of transgenes in episomal vectors $(20,21)$. In contrast, retrovirus and lentivirus allow long-term transgene expression by random integration into the host genome. However, these vectors tend to be inefficient at transducing primary B cells $(22,23)$. In the few examples of successful reprogramming of primary B cells, genetically modified B cell have been applied for presentation of recombinant antigen for inhibition of immunity in a mouse model of multiple sclerosis (24) or induction of tolerance toward therapeutic proteins (25). The revolutionary advances in targeted genome editing has paved the way for alternative strategies to genetically modify immune cells (26-28). So far, the CRISPR-Cas9 system has been mainly applied to integrate transgenes into lymphomaderived or hybridoma cell lines by homology-directed repair (HDR) (29-31). Precise genome editing in primary murine B cells derived from murine transgenic models endogenously expressing Cas9 protein showed efficient gene disruption based on nonhomolgous end-joining (NHEJ) repairs (32). Furthermore, a few recent studies used CRISPR-Cas9 for site-specific gene disruption or transgene integration by HDR in human primary B cells $(19,33,34)$. Hung et al. demonstrated that delivery of Cas9 ribonucleoprotein (RNP) complexes in combination with HDR DNA templates enabled the engineering of plasma cells to secrete therapeutic proteins. This proposes the attractive prospect of establishing a controllable system in which exposure to antigen can induce engineered B cells that produce therapeutic proteins.

Establishing a preclinical genome editing platform based on primary murine $\mathrm{B}$ cells enables the investigation of these cells as novel vehicle for adoptive immune cell therapies. In the present study, we have molecularly designed and optimized a novel class of synthetic antigen receptors, chimeric B cell receptors (CBCR), which were stably integrated by CRISPR-Cas9 into immortalized and primary murine B cells. First, we assess the stable expression of a broad range of constructs encoding a model antigen-specific CBCR linked to a green fluorescent protein (GFP) reporter in B cell hybridoma line. We genomically modify B cells by targeting a safe harbor locus (Rosa26) with CRISPR-Cas9 RNP complexes and CBCR HDR templates in the form of dsDNA. We then optimize CBCR expression and detection by a series of modification to the extracellular surface tag, transmembrane domain, and intracellular signaling domains. Based on the results obtained from construct screening in hybridoma cells, selected constructs displaying high levels of surface expression are further evaluated in murine primary B cells. Collectively, we could achieve the precise integration of CBCRs into the Rosa26 locus of primary murine B cells, its surface expression and selective enrichment of engineered cells. In the future, CBCR engineered B cells can be evaluated in preclinical in vivo models in order to assess their potential in versatile immune cell therapy applications.

\section{RESULTS}

\section{Design of Chimeric B Cell Receptors (CBCRs)}

In this study, we aimed to create a chimeric B cell receptor that is able to recognize antigen independently of the endogenously expressed B cell receptor. We initially used immortalized B cell hybridomas to screen a broad range of CBCR constructs encoding an antigen-binding domain, a spacer region that includes a detection tag, a transmembrane and cytoplasmic signaling domains (Figure 1A). For each of these constructs we generated a stable cell line by CRISPR-Cas9 mediated integration of the transgene cassette into the safe harbor locus Rosa26, which has been validated to stably express robust levels of the transgenes, while minimizing proximity to proto-oncogenes and adverse effects on the host cell. Here, we used a parental hybridoma cell line which constitutively expresses Cas9 from the Rosa26 safe harbor locus approximately $6 \mathrm{~kb}$ downstream of the CBCR integration site, as it permits the transfection of just pre-formed gRNA and ssODN (30). Additionally, this original cell line, that will be referred to as HC9-, contains a frameshift mutation in its endogenous antibody variable heavy chain region, resulting in the knockout of antibody expression.

Within the $5^{\prime}$ portion of the Rosa26 locus, we identified several potential gRNA sites compatible with S. Pyogenes Cas9 and its protospacer adjacent motif (PAM, 5'-NGG). The activity of Cas9 at each gRNA site in B cells was evaluated by measuring non-homologous end-joining (NHEJ) via surveyor nuclease assay (Figure S1). The gRNA with the highest activity (gRNA1) was selected for all subsequent genome editing experiments (Figure 1B). To precisely integrate the CBCR by Cas9-mediated HDR, donor templates were designed including the respective CBCR transgene flanked by homology arms of $1.4 \mathrm{~kb} / 1.5 \mathrm{~kb}$ 


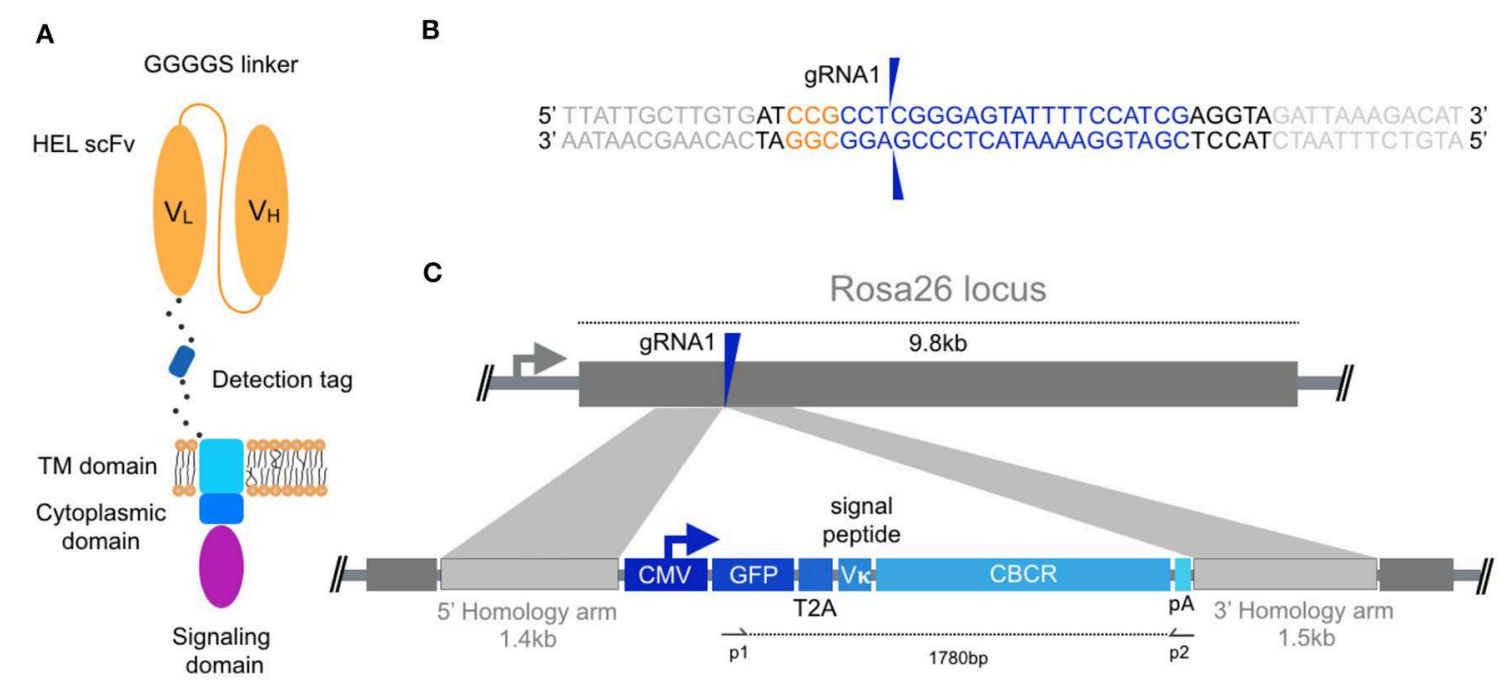

B
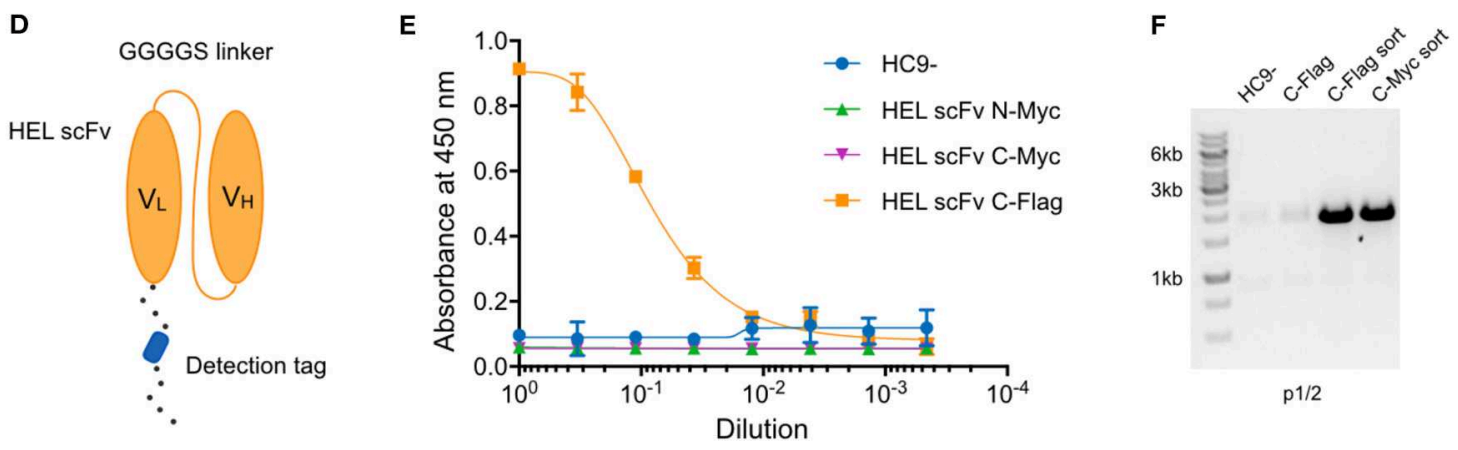

FIGURE 1 | Genomic integration of chimeric B cell receptors (CBCR) by CRISPR-Cas9-mediated HDR. (A) Schematic shows the design of the CBCR, which consists of an scFv-based antigen recognition domain (specific for a hen-egg lysozyme [HEL]) (orange), an extracellular spacer with a detection tag, a transmembrane (TM) domain (light blue), and endodomains (blue, purple). scFV, single chain variable fragment; VL, light chain variable domain; VH, heavy chain variable domain. (B) Shown is the gRNA sequence (blue) for Cas9-targeting of the safe-harbor locus Rosa26 locus; also shown is the corresponding PAM sequence (orange) and the beginning of the homology arms in the HDR template (light gray). The two blue arrows indicate the predicted Cas9 double-stranded break site. (C) CRISPR-Cas9-mediated HDR for genomic integration of CBCR construct into the Rosa26 locus. The PCR-linearized donor template contains a GFP reporter gene followed by a T2A coding sequence, the $V_{\kappa}$ signal peptide, the CBCR cassette and a poly-A sequence all under the control of a CMV promoter and flanked by sequences homologous to the Rosa26 locus next to the gRNA target site of 1.4 and $1.5 \mathrm{~kb}$, respectively (light purple). T2A: the self-cleaving thosea asigna virus 2A sequence, pA: SV40 polyA sequence. (D) Schematic shows the format of the CBCR antigen binding domain including a linker with a detection tag, either Myc or Flag epitope. (E) Cells were enriched for GFP ( $488 \mathrm{~nm}$ ) expression via FACS. Graph shows ELISA results of scFv secretion levels (capture HEL antigen, detection anti-Myc, or Flag) on enriched hybridoma culture supernatant for scFv variants (excluding the TM and intracellular domains shown in $\mathbf{D}$ ) with Myc or Flag detection tag in N-or C-terminal position. Supernatant of HC9- cells was used as negative control. For each sample, three technical replicates were analyzed and a four-parameter logistical curve was fitted to the data by non-linear regression. Data are presented as the mean and error bars indicate standard deviation. (F) RT-PCR on mRNA of scFv variants with C-terminal detection tags was performed with primers shown in c displaying expression of the transgenic scFv cassette after transfection only and transfection followed by sorting on GFP expression.

consistent to the Rosa26 sequences next to the gRNA1 target site (Figure 1C). The PAM was not incorporated in the repair template, so that the repaired sequence would not to be cleaved by Cas9. The full transgene consisted of a $V_{\kappa}$ leader sequence, a GFP reporter gene followed by a self-cleaving T2A sequence, and the $\mathrm{CBCR}$ all under the control of the cytomegalovirus (CMV) promoter. The HDR donor was generated by PCR to obtain a linearized format and electroporated in combination with gRNA1 into the HC9- hybridoma cells. At $\sim 72 \mathrm{~h}$ post-transfections, $\mathrm{GFP}^{+}$cells were isolated by fluorescenceactivated cell sorting (FACS) and expanded in culture.
For the extracellular antigen-binding domain, we used scFvs, as these have been successfully used in previously engineered receptors such as CARs and synNotch (10, 35). Additionally, scFvs offer great stability and high-affinity ligand binding (36). As a target, we selected the model protein hen egg lysozyme (HEL) due to its small size, easy availability and the presence of valuable research tools such as a HEL-specific B cell transgenic mouse model and well-described HEL-specific antibodies and scFvs (37-39). Initially, we designed an scFv of the highaffinity antibody HyHEL10 in the $\mathrm{V}_{\mathrm{L}}-\mathrm{V}_{\mathrm{H}}$ orientation. After comparison of this $\mathrm{scFv}$ and the affinity-improved M3 mutant 
scFv derived from the anti-HEL antibody D1.3, we proceeded with the M3 scFv due to increased detection signal by enzymelinked immunoabsorbent assays (ELISA) (Figure S2) (40). While CBCR expression can be detected by antigen labeling, this is often accompanied by B cell activation, therefore an orthogonal detection method would be valuable. While the GFP offers a selection marker for integration, it does not directly indicate surface expression of CBCR, thus the tactical introduction of a detection tag provides another identification marker. As has been previously shown with CARS in T cells, careful design of tag sequences and its location is required (41-43). Initially the M3 scFv was equipped with an N-terminal Myc epitope or fused to a C-terminal spacer sequence (26aa) incorporating a Myc or Flag epitope (Figure 1D). We used a secretion variant of the CBCR, which lacks the transmembrane and intracellular signaling domains to evaluate integration and secretion levels of HEL-binding scFv. We used enzyme-linked immunoabsorbent assays (ELISA) on normalized culture supernatants. Drastic improvement in scFv secretion was observed for cells in which the Flag sequence was introduced into the C-terminal spacer as compared to both Myc epitope containing variants (Figure 1E), whereas equal RNA expression levels were confirmed by RT-PCR (Figure 1F) indicating impairment of either proper scFv protein folding or secretion by the Myc epitope tag. RNA expression of Cterminally incorporated Flag tag was significantly increased after enrichment for GFP+ cells via FACS compared to unsorted cells (Figure 1F).

\section{Optimization of CBCR for Robust Surface Expression on Hybridoma B Cells}

Next, we investigated whether a CBCR with the previously characterized scFv domain was presented at the cell surface, while maintaining antigen HEL binding. For this purpose, we linked the $s c F v$ clone M3 and the spacer incorporating the Flag epitope sequence (DYKDDDDK) to the transmembrane domain (TM) and the short cytoplasmic domain of the endogenous murine BCR (IgG2c) referred to as CBCR-BCR-TM. Alternatively, the spacer was fused to a CD28 transmembrane domain (CBCRCD28-TM), which has been successfully incorporated in the design of several CARs (44) (Figure 2A). When expressed in HC9- hybridoma cells, both CBCR-BCR-TM and CBCR-CD28TM were detected on the cell surface based on HEL antigen binding for cells enriched for GFP by FACS (Figure 2B, upper row). CBCR-CD28-TM was expressed at the cell surface to a substantially greater extent than CBCR-BCR-TM. Additionally, CBCR cells were stained with anti-Flag antibody. The CBCR surface expression demonstrated by HEL antigen recognition in $\mathrm{GFP}^{+}$cells does not correlate with the CBCR detection via the Flag peptide tag, suggesting impaired accessibility of the Flag epitope, once the spacer is fused to a transmembrane domain (Figure 2B, lower row). However, we were able to identify clones demonstrating both a clear Flag tag expression and HEL antigen binding after preforming single-cell sorting and expansion (Figure 2C, right). While revealing a similar level of stable GFP expression (Figure 2C, left), CBCR-CD28$\mathrm{TM}$ expression was increased compared to CBCR-BCR-TM expression on the surface, consistent with the data of bulk sorted cells based on HEL binding only (Figure 2B). To evaluate stable and targeted integration of the CBCR cassette on a genotypic level, PCR assays on genomic DNA were designed (Figure 2D). The introduced cassette was detected by PCR in the single-cell line expressing CBCR-CD28-TM, but not in the parental HC9cell line (p3/4). PCR analysis showed the presence of at least one residual wildtype allele in the cell line (Figure 2E). Genomic PCR using primers $\mathrm{p} 5$ and $\mathrm{p} 6$ confirmed precise integration of the CBCR gene into the Rosa26 locus (Figure 2F).

\section{Strep Tag II Incorporation Improves CBCR Surface Expression and Selection}

Previous studies have reported that the length of the nonsignaling extracellular spacer can have an impact on surface expression or receptor activity $(37,43)$. We constructed variants with shorter and extended spacers in combination with both TM domains in order to analyze the influence on surface expression of the CBCR and the accessibility of the Flag detection tag within the spacer. HEL antigen binding within the $\mathrm{GFP}^{+}$population was examined for cells expressing CBCR including spacer regions of different lengths before and after sorting of $\mathrm{GFP}^{+}$cells (Figures 3A,B). Antigen binding did not vary significantly with extracellular linker length, indicating that the composition of the spacer does not affect the surface expression in these cases. Detection of the Flag tag in $\mathrm{GFP}^{+}$bulk-sorted cells still was not improved after modifying the length of the spacer (Figure 3C). To address the tag detection, we introduced one or more Strep tag II sequences replacing the Flag epitope within the Gly/Ser spacer (Figure 3D). All Strep tag CBCR were stained with antiStrep tag II antibody after enrichment of $\mathrm{GFP}^{+}$cells via FACS. Staining intensity was significantly increased for both CBCR-TM variants with one Strep tag II compared to the signal provided by the single Flag epitope (Figure 3E). Surface expression based on antigen binding and tag detection again revealed increased level for CBCR-CD28-TM compared to the CBCR-BCR-TM variant. Further, staining intensity was dramatically enhanced for CBCR-CD28-TM cells that contained three Strep tag II sequences (Figure 3E, right). These data indicate that inclusion of Strep tag II improves the CBCR surface expression and its correlation with staining based on the detection tag.

\section{Incorporation of a CD79 $\beta$ Signaling Domain Improves CBCR Surface Expression}

In order to generate a functional receptor, an intracellular signaling domain is required for signal transduction. As the endodomain of surface-bound immunoglobulins (Ig) itself is very short und incapable of intracellular signal transmission, the endogenous BCR only functions as a complex composed of the Ig molecule associated with a heterodimer called Ig- $\alpha / \operatorname{Ig}-\beta$ or $\mathrm{CD} 79 \alpha / \beta$. The CD79 subunits contain an immunoreceptor tyrosine-based activation motif (ITAM) in their cytoplasmic domains, which recruit Syk tyrosine kinase and mediate $B$ cell activation upon antigen binding and subsequent phosphorylation $(45,46)$. Previous work suggested that $\mathrm{CD} 79 \alpha$ and $\mathrm{CD} 79 \beta$ are independently sufficient to trigger protein 


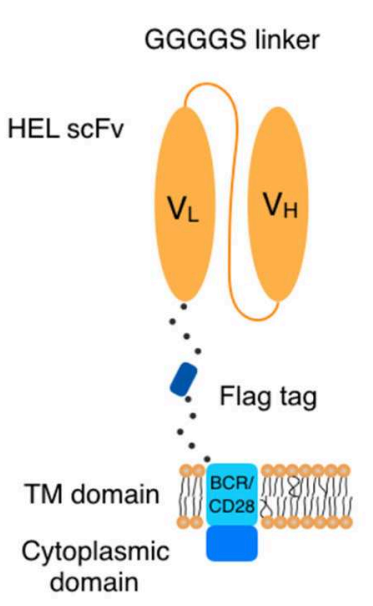

B

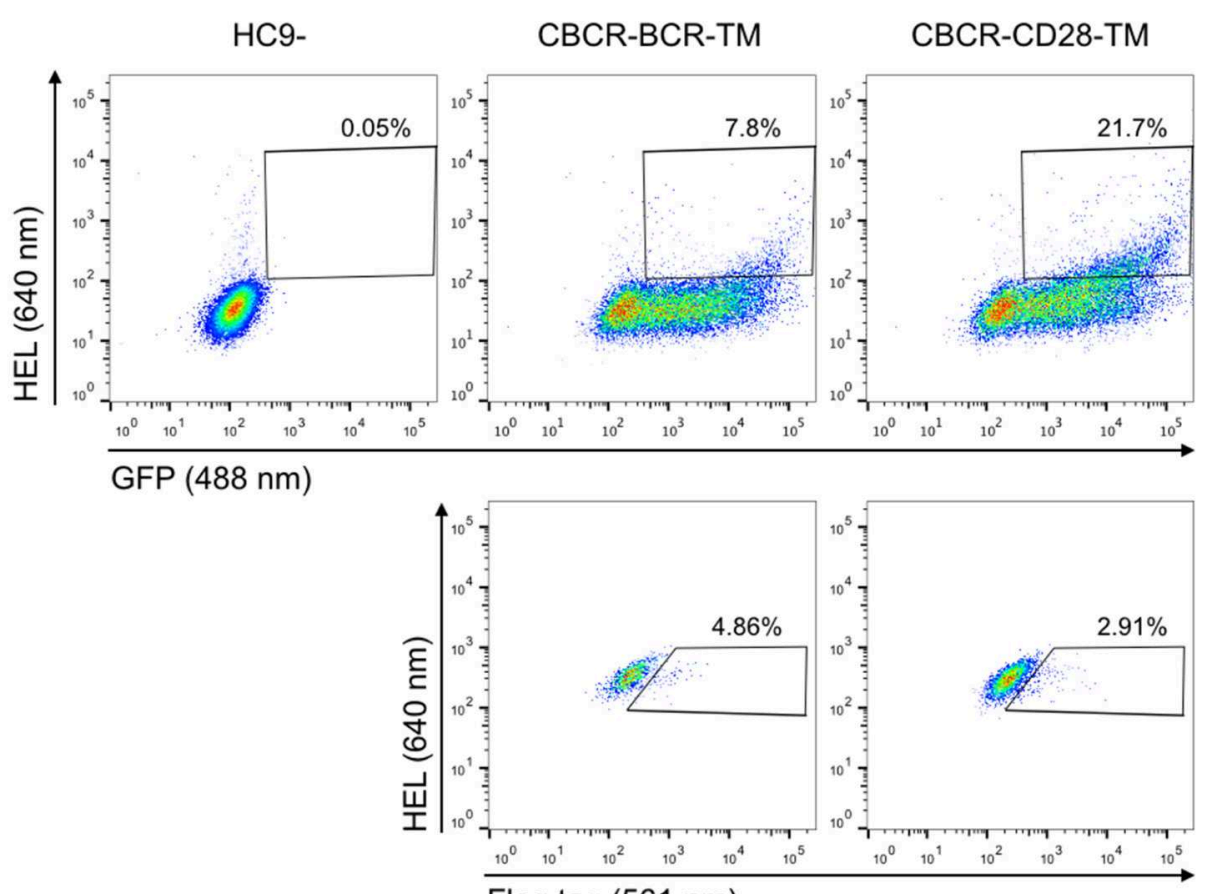

Flag tag (561 nm)
C

HC9- CBCR-BCR-TM

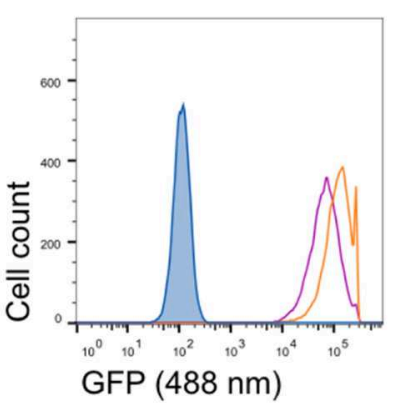

CBCR-CD28-TM

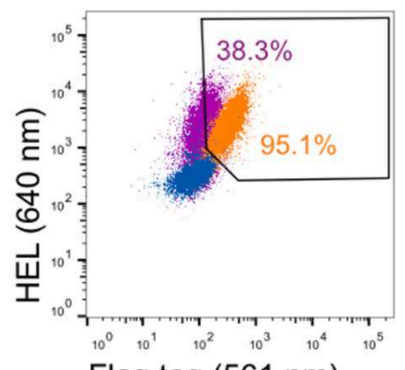

Flag tag (561 nm)
E

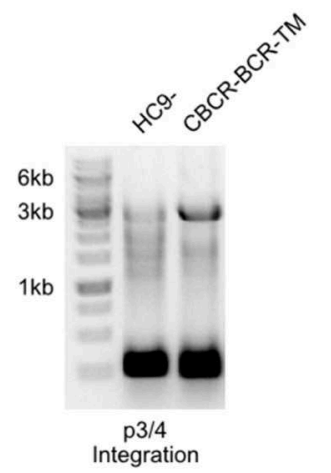

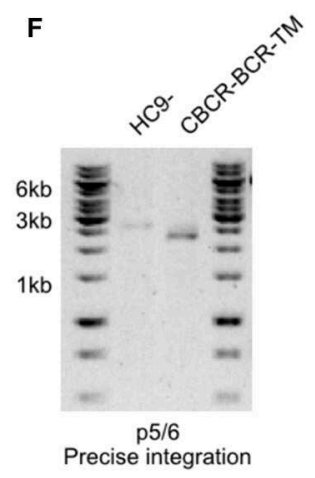

D

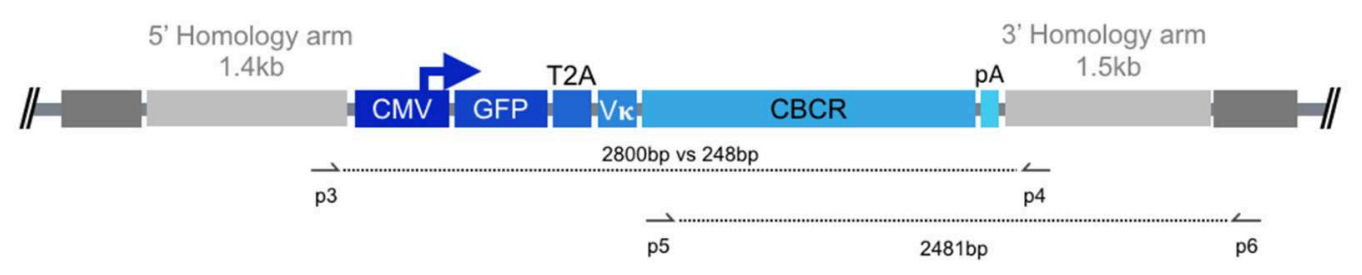

FIGURE 2 | Stable surface expression of CBCR on hybridoma cells. (A) Schematic of surface expressed CBCR including a Flag detection tag and varying transmembrane (TM) domains. The TM domain is derived from either the endogenous BCR composed of an immunoglobulin (purple in $\mathbf{C}$ ) or the T cell costimulatory CD28 molecule (orange in C). (B) Representative flow cytometry dot plots of the cells after enrichment based on GFP reveal CBCR surface expression (via HEL antigen binding) for both variants (upper row), but no correlation with surface detection via the Flag protein tag (561 nm, lower row). The parental hybridoma cells were used as negative control. Cells that were positive for HEL binding and Flag tag expression were enriched by FACS. (C) Flow cytometry analysis of resulting single-cell clones selected for GFP expression and binding to HEL shows comparable levels of persistent GFP expression for both construct variants (left), but differs in terms of CBCR surface expression (right). (D) Schematic of primer sets for genomic DNA analysis in order to detect transgene integration (p3/4) and to confirm GFP-2A-CBCR cassette integration at the correct Rosa26 locus (p5/6). (E) Agarose gel shows genomic PCR products that confirm the presence of the transgene in the cell line expressing the $\mathrm{CBCR}$ containing the $\mathrm{BCR}$ transmembrane $(2,800 \mathrm{bp})$. Additionally, the presence of at least one wt allele is demonstrated by the PCR product with a length of $248 \mathrm{bp}$. (F) Genomic PCR analysis verifies the integration of the GFP-T2A-CBCR-BCR-TM cassette in the correct locus (2,481 bp) in the same cell line as in (E). PCR products in $(\mathbf{E}, \mathbf{F})$ were verified by Sanger sequencing. 
A

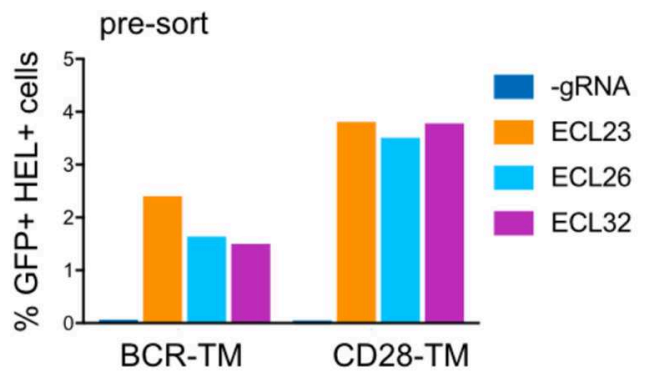

C

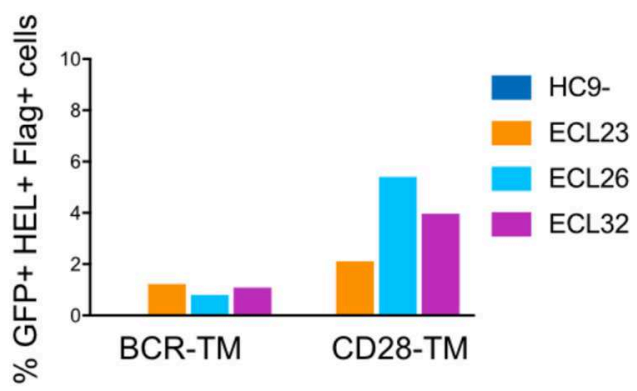

B

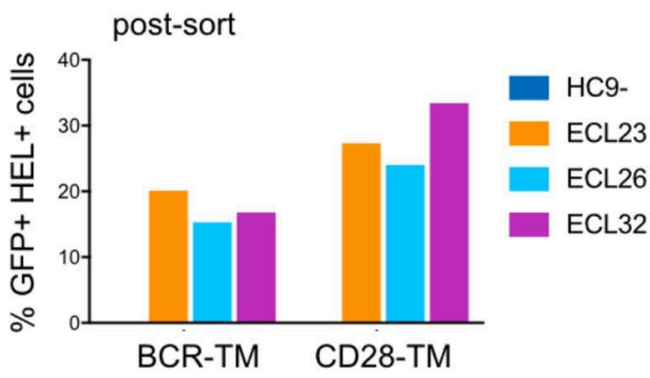

D

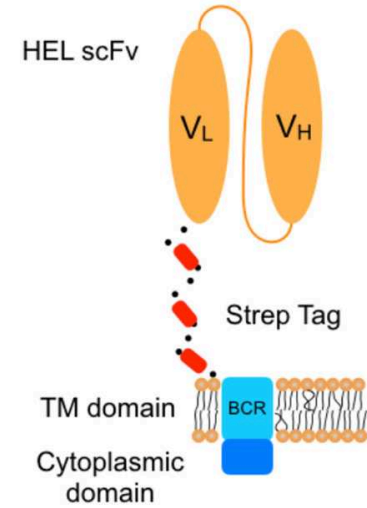

E

HC9-

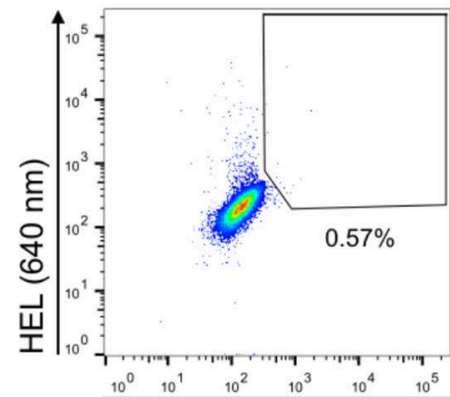

CBCR-BCR-TM

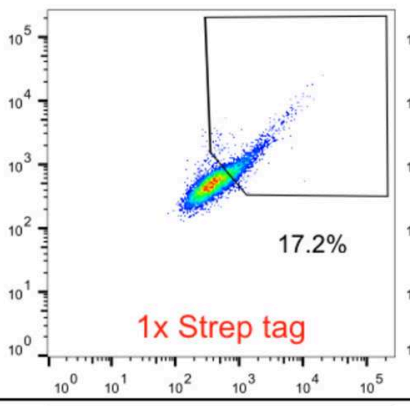

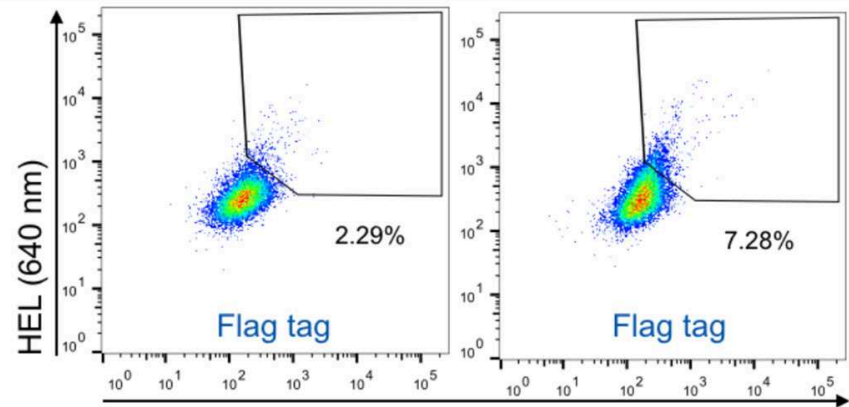

Flag tag $(561 \mathrm{~nm})$

\section{CBCR-CD28-TM}

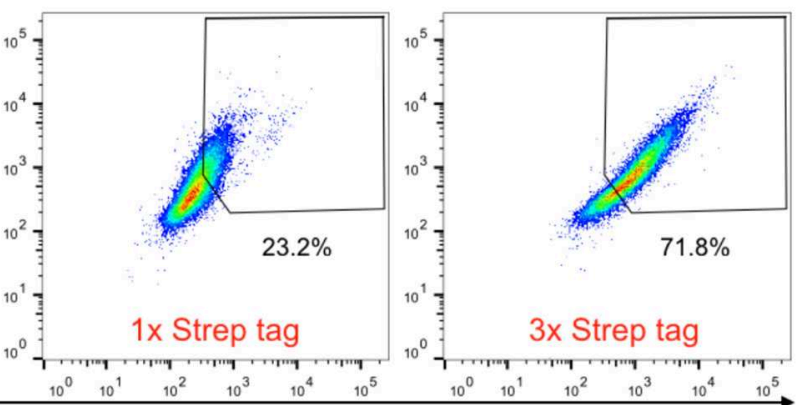

FIGURE 3 | Optimization of CBCR receptor design to improve surface expression and the detection of surface presentation via tag. (A) The surface expression in hybridomas of representative CBCR constructs was evaluated by flow cytometry before (A) and after (B) enrichment based on GFP expression (488 nm) on day 3 after transfection. Variants shown differ in the transmembrane (TM) domain and the lengths of the extracellular linkers (ECL). (C) Bar graph shows percentage of cells detectable via Flag tag within the population (B) of cells showing GFP ${ }^{+}$expression and HEL binding after sorting. (D) Schematic of CBCR design shows replacement of Flag epitope with a triple Strep epitope II tag (red). (E) CBCR surface expression of GFP sorted cells (488 nm) was quantified by flow cytometry based on Strep II tag detection ( $405 \mathrm{~nm}$ ) using variants that incorporate a single or triplet Strep epitope (upper row). Antigen recognition was additionally confirmed by HEL binding $(640 \mathrm{~nm})$. Representative flow cytometry plots show percentages of HEL binding and Flag ${ }^{+}(561 \mathrm{~nm})$ cells for the CBCR variants including the single Flag tag after GFP sorting as a control (lower row). 
tyrosine kinase activation and induction of downstream signaling cascades, as long as the ITAM regions remain intact (47). Here, we fused the complete intracellular domain of either the CD79 $\alpha$ or CD79 $\beta$ polypeptide C-terminally to the short cytoplasmic tail of the Ig molecule (Figure 4A). HC9- cells were transfected with both constructs as previously described and stable CBCR expression based on HEL binding and Strep tag II detection was analyzed using flow cytometry after enrichment for $\mathrm{GFP}^{+}$cells. CBCR expression was substantially increased for cells expressing CBCR-CD79 $\beta$ compared to CBCR-CD79 $\alpha$, which was even higher compared to previous constructs without intracellular signaling units (Figure 3E, upper row). This suggests that improved expression or cell-surface transport for the CBCR variant occurs when incorporating the $C D 79 \beta$ signaling domain (Figure 4B). This higher CBCR expression was consistent across several selected single-cell clones (Figure 4C, right), while GFP expression was comparable, but intensity was slightly increased for CBCR-CD79 $\beta$ expressing cells (Figure 4C, left). Genomic $\mathrm{PCR}$ analysis using primers $\mathrm{p} 7$ and $\mathrm{p} 8$ verified precise integration of the GFP-CBCR-CD79 $\beta$ cassette into the Rosa26 locus one of the selected single-cell clones (Figures 4D,E). Conclusively, incorporation of a signaling domain did not interfere with CBCR surface expression.

\section{Evaluating HDR Protocols for Primary Murine B Cells}

Because of the capacity to select and expand immortalized hybridoma cells, high genome editing efficiencies are not essential. However, with primary B cells optimized and high HDR efficiencies are very important. We first isolated murine splenic B cells and cultured them using an in vitro expansion system based on $40 \mathrm{LB}$ feeder cells (NIH3/T3 fibroblasts that stably express BAFF and CD40 ligand) (48) in the presence of IL-4 up to 4 days for pre-activation (Figures $5 \mathbf{A}, \mathbf{C}$ ). Next, we electroporated Cas9-guide RNP complexes together with $5 \mu \mathrm{g}$ of PCR-linearized HDR donor template into primary B cells (Amaxa nucleofection). Three days after transfection, cells were analyzed and enriched for GFP expression by FACS. Sorted cells were cultured for an additional 6 days under activating conditions by replacing IL- 4 with IL-21. CBCR surface expression was determined via flow cytometry and genomic DNA analysis was performed at Day 10 to confirm precise integration into the Rosa26 locus (Figure 5A). Expansion on $40 \mathrm{LB}$ feeder cells was only negligibly affected by transfection of Cas9-RNP and dsDNA as compared to non-transfected cells co-cultured in presence of IL-4 (Figure 5B). Primary B cells cultured in the presence of soluble BAFF and IL-4 showed only minor overall increase in the number of live $B$ cells. (Figure 5B). Subsequently, the influence of pre-activation on transfection efficiencies was determined. For this purpose, we transfected $10^{6}$ primary B cells with $2 \mu \mathrm{g}$ plasmid DNA encoding for a CMV promoter-driven GFP reporter gene directly after isolation from a mouse spleen or following pre-activation on $40 \mathrm{LB}$ feeder cells for $1,2,3$, or 4 days and analyzed GFP expression by flow cytometry $24 \mathrm{~h}$ after transfection. We observed enhanced transfection efficiencies and viability after pre-activation compared to transfection of freshly isolated B cells, consistent with previous findings in primary T cells (49). The highest transfection efficiency was observed after 1 day of pre-activation, followed by 4 days of pre-activation, suggesting a correlation with the time points of high proliferation rates. Applying a protocol including 1 day of pre-activation and delivery of components previously optimized in hybridoma cells only revealed very small numbers of precisely edited primary cells identified by GFP expression (0.1-0.3\%).

Several studies suggest that the use of single-stranded (ss) DNA increases the frequency of HDR, most notably through the use of adeno-associated virus (AAV) (30, 50, 51). AAV can package at least $4.9 \mathrm{~kb}$ offering an HDR donor template compatible with CBCR and homology arms. Previous studies have reported relatively high levels of AAV-mediated HDR in multiple cell types, including $\mathrm{T}$ lymphocytes (52-54). To investigate AAV transduction efficiency in primary murine $\mathrm{B}$ cells, we screened several AAV serotypes possessing a reporter GFP gene (Figure 5D). We transduced B cells after preactivation on $40 \mathrm{LB}$ for 1 day and analyzed transient GFP expression by flow cytometry after 3 days, which represented the overall transduction efficiency. The highest transduction efficiency was achieved with the synthetic AAV-DJ serotype (Figure 5D). Regardless of serotype, we observed minimal loss in cell viability after exposure to the virus particles. Next, we examined the frequency of HDR-mediated integration of a larger-size transgene delivered by recombinant AAV-DJ. For this purpose, an HDR donor cassette consisting of a CMV-driven GFP reporter gene was designed with homology arms of 750 bp in size to meet AAV payload restrictions (Figure 5E). After pre-activation and electroporation with or without complete Cas9-RNP, B cells were transduced with AAV-DJ CMV-GFP at various multiplicity of infections (MOIs) and cultured for an additional 6 days in the presence of 40 LB cells and IL-21 (Figures 5F,G). We observed only minor loss in viability, even at the highest AAV dose. Approximately $3-4 \%$ of cells that were treated with AAV alone (MOI $1 \times 10^{5}$ ) showed persistent GFP expression implying a relatively high background of episomal expression (Figure 5F). In cells that received both AAV-DJ delivered HDR donor and Cas9-RNP (targeting the Rosa26) we observed only a marginal increase by HDR (1.2-1.5-fold), measured by stable GFP expression (Figure 5G). While HDR efficiencies in primary $B$ cells were only marginally enhanced by AAV-DJ delivered HDR donor, HDR-mediated integration efficiencies were dramatically improved in hybridoma cells, suggesting that primary B cells may have inherent limitations in HDR processes (Figure S3). When directly compared to dsDNA, we found that AAV-DJ showed slightly improved HDR-mediated integration, however it also resulted in strong background GFP expression complicating the discrimination of precisely edited cells especially considering the limited life span and restrictions in selection of primary $\mathrm{B}$ cells in in vitro culture (Figure 5G). The results described here suggest that despite the relatively low HDR-efficiencies, dsDNA HDR template is more suitable for CRISPR-Cas9-mediated genome editing in primary B cells due to its reliable discrimination of successfully modified cells. 
A

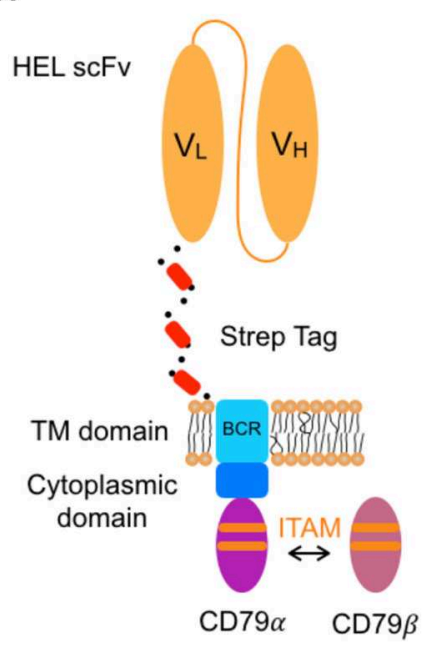

B

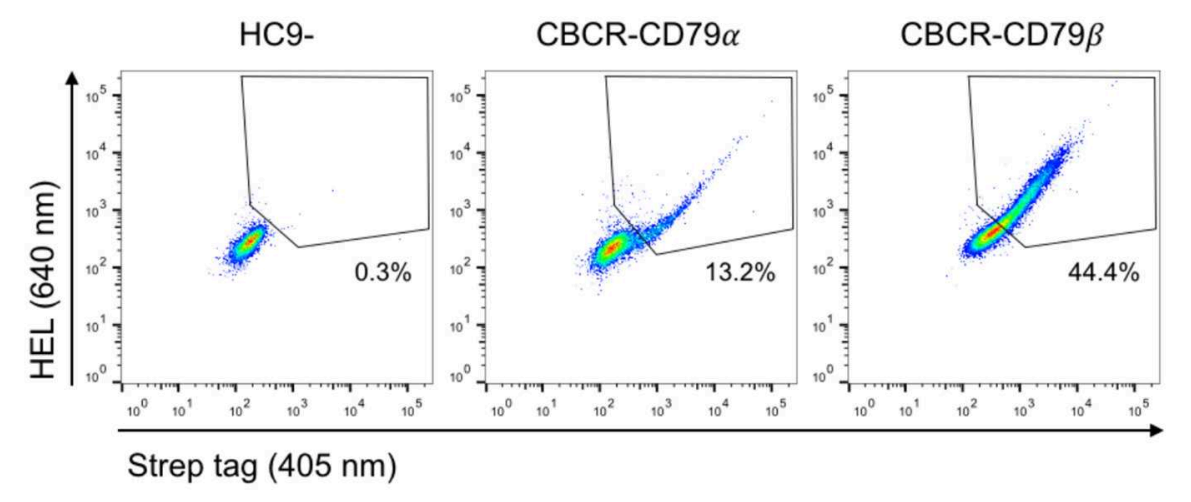

D

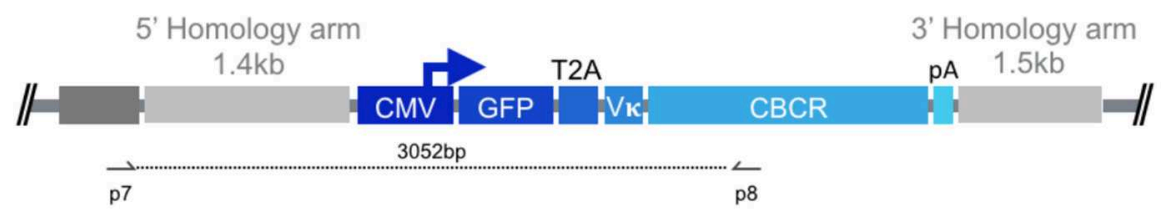

C

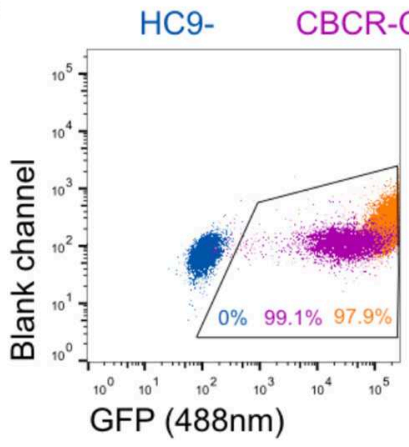

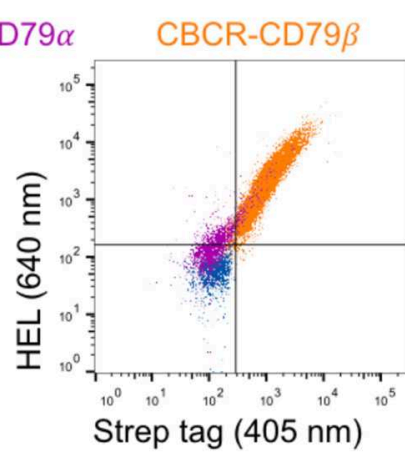

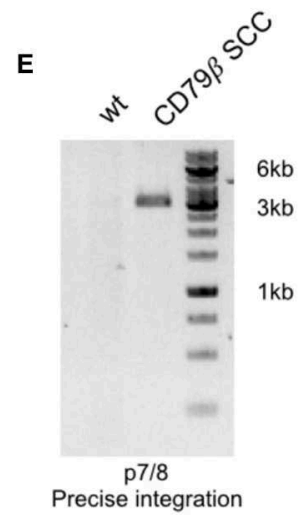

FIGURE 4 | Improved surface expression of CBCR with CD79 $\beta$ signaling domain. (A) Schematic of the CBCR complex containing the intracellular domain of either a CD79 $\alpha$ or CD79 $\beta$ transmembrane protein C-terminally fused to the cytoplasmic BCR domain. (B) Representative flow cytometry dot plots show hybridomas with stable CBCR surface expression based on HEL binding $(640 \mathrm{~nm})$ and Strep II tag detection $(405 \mathrm{~nm})$ for GFP enriched cells having a CBCR with either the CD79 $\alpha$ or CD79 $\beta$ intracellular domain precisely integrated. Data are representative for three independent experiments. (C) Flow cytometry dot plots show GFP expression (left) and HEL binding and Strep II tag detection of exemplary single-cell clones following sorting ( $\mathrm{HEL}^{+}$Strep ${ }^{+}$) on samples in b. Tendency of decreased surface expression for the CBCR with the CD79 $\beta$ intracellular domain was validated in multiple single-cell clones. (D) Schematic of primer set for genomic DNA analysis in order to confirm integration of GFP-2A-CBCR cassette at the correct locus (p7/8). (E) Genomic PCR analysis verifies the integration of the GFP-T2A-CBCR-CD79 $\beta$ cassette in the correct locus of a single-cell clone (SCC, 3,052 bp). The band was extracted and Sanger sequencing confirmed the precise integration in the Rosa26 locus.

\section{Robust CBCR Genomic Integration and Surface Expression of CBCR in Primary B Cells}

We evaluated the surface expression of the previously optimized CBCR variants in order to generate primary B cells capable of antigen recognition independent of their endogenously expressed BCR. For this purpose, we transfected pre-activated B cells with Cas9-RNP targeting the Rosa26 locus and PCR-derived HDR donor (GFP-T2A-CBCR-CD79 $\alpha / \beta$ and GFP-T2A-CBCRBCRTM/CD28TM) and recovered them on $40 \mathrm{LB}$ feeder cells in the presence of IL-4. At day three after electroporation, we observed low, but robust HDR-mediated integration levels, measured by persistent GFP expression, compared to a negative control of cells receiving PCR-linearized repair template and Cas9 protein without gRNA (Figure 6A). $\mathrm{GFP}^{+}$cells were enriched via FACS, expanded in the presence of IL-21 and analyzed by flow cytometry for CBCR surface expression based on HEL antigen binding and Strep tag II detection on day 10 (Figure 6B). We found substantial enrichment for GFP expressing cells, from which a robust fraction is expressing either CBCR variant, thus, indicating that CBCR expression is tolerated in primary $B$ cells. HEL antigen binding by the CBCR does not appear to be inhibited by expression of native BCR. Similar to our observations in hybridoma B cells, CBCR detection based on the 
A
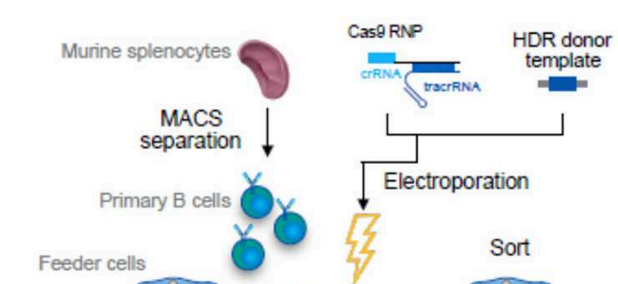

Culture time (d)

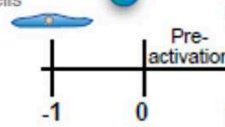

Electroporation
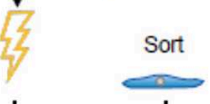

$\prod_{1}$

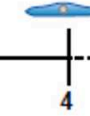

西

C

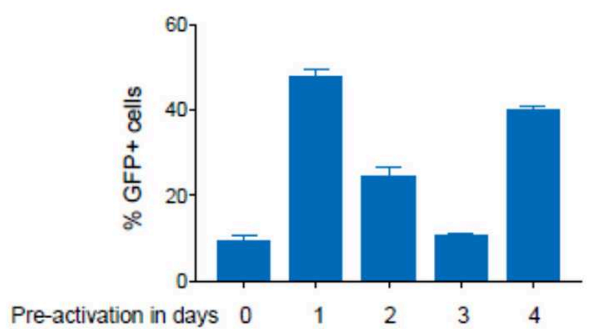

D

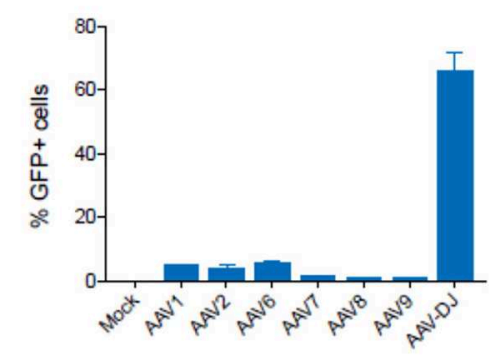

E

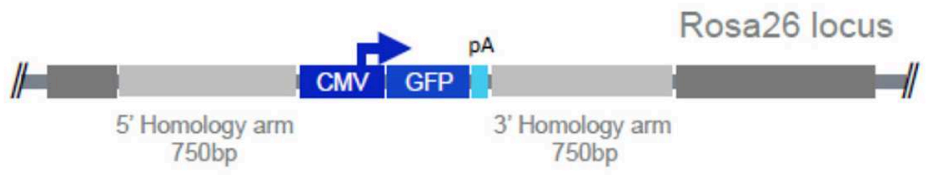

F

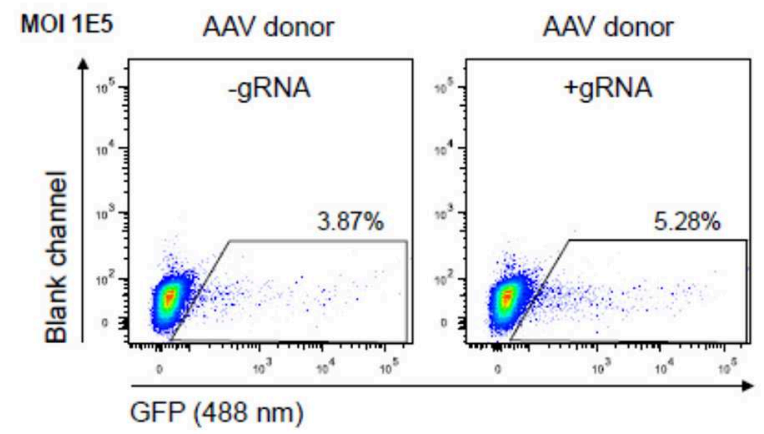

B

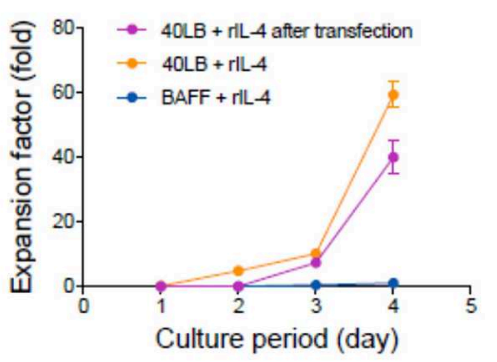

Culture period (day)

Pre-activation in days $\begin{array}{lllll}0 & 1 & 2 & 3 & 4\end{array}$

10

G

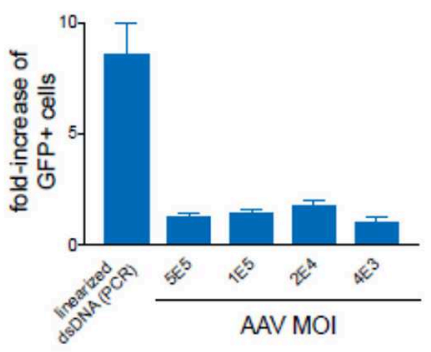

FIGURE 5 | Targeted genome editing in primary murine B cells using CRISPR-Cas9. (A) Overview shows timeline of Cas9-gRNA RNP delivery to primary B cells isolated from murine spleen. B cells were initially co-cultured with feeder cells before and after transfection of RNPs and HDR donor template DNA on day 1. Three days after transfection cells were enriched for GFP expression via FACS and analyzed at Day 10 after expansion. (B) Cumulative fold increase in the number of live B cells cultured in the following conditions: (i) in presence of soluble BAFF and IL-4; (ii) on 40 LB in the presence of IL-4, (iii) or on 40 LB in the presence of IL-4 and after electroporation with Cas9-RNP and PCR-linearized double-stranded (ds) repair template DNA after pre-activation on 40 LB for 1 day. (C) $10^{6}$ primary B cells were transfected with $2 \mu \mathrm{g}$ plasmid DNA (pMax-GFP) directly after isolation from mouse spleen or following pre-activation on 40 LB feeder cells for 1, 2, 3, or 4 days. Data show the percentage of GFP expressing cells determined by flow cytometry $24 \mathrm{~h}$ after transfection. Data are presented as the mean and error bars indicate standard deviation $(n=2)$. (D) Splenic B cells were pre-activated for 1 day and were either mock treated or transduced with GFP-expressing sSAAV using a comprehensive panel of AAV serotypes $\left(1,2,6,7,8,9\right.$, or DJ) at a MOI of $10^{5}$. The bar plot shows the percentages of GFP ${ }^{+}$cells after $72 \mathrm{~h}$ ( $n=3,3$ independent experiments). (E) Schematic of the HDR donor cassette encoding for the GFP reporter gene with 750 bp flanking homology arms after integration into the Rosa26 locus. (F) After one day of pre-activation on $40 \mathrm{LB}$, primary B cells were transfected with Cas9-RNP immediately followed by HDR repair template delivery via chimeric AAV serotype DJ encoding the GFP reporter gene. Representative flow cytometry dot plots show GFP expression $(488 \mathrm{~nm})$ day 10 after genome editing for transduction with a MOI of $1 \times 10^{5}$. Cells transfected only with Cas9-protein without gRNA and transduced with GFP expressing HDR donor packaged using scAAV-DJ were used as negative control to determine the level of GFP expression from episomal retention. (G) Data are displayed as fold-increase of AAV-DJ transduced GFP+ cells receiving the Cas9-gRNA complex to cells transfected with Cas9-protein only representing the HDR based integration. Cells transfected with Cas9-protein only indicate the episomal AAV background expression. Cells transfected with Cas9-RNP and PCR-linearized dsDNA served as control. GFP expression was measured on day 9 after transfection. All data are means \pm s.d $(n=3)$. 
Flag tag was only weak (Figure 6B and Figure S4A). Consistent with hybridomas, we also observed an enhanced CBCR-CD79 $\beta$ expression as to CBCR-CD79 $\alpha$ was confirmed in primary $\mathrm{B}$ cells. PCR analysis on genomic DNA extracted from primary $B$ cells with or without FACS mediated enrichment verified the targeted integration of the GFP-T2A-CBCR cassette into the Rosa26 locus (Figure 6C and Figure S4B). Taken together, these findings demonstrate that we have developed a reliable and consistent pipeline to precisely introduce cassettes of several $\mathrm{kb}$ size into the genome of primary murine B cells using CRISPRCas9 induced HDR. Furthermore, we were able to show the robust surface expression of a synthetic, antigen-specific CBCR in primary B cells.

\section{DISCUSSION}

Immune cell therapies based on the integration of synthetic antigen receptors comprise a successful and rapidly expanding therapeutic option for the treatment of cancer, most notably CAR expressing $\mathrm{T}$ cell therapies $(1,35,43)$. Additional to existing $\mathrm{T}$ cell therapies, B lymphocytes hold promise as novel donor cells for adoptive cell therapies due to their natural properties, such as longevity, and immense protein secretory capacity $(18,19)$. Here, we have demonstrated targeted genomic integration in murine $\mathrm{B}$ cells of a novel class of synthetic antigen receptorsCBCR. CBCRs offer a potential way to activate and expand engineered $B$ cells in antigen-controllable manner, independent of the endogenously expressed BCR.

We designed CBCR constructs to encode an antigenbinding domain consisting of an $\mathrm{scFv}$, a spacer region that includes a detection tag, a transmembrane domain and cytoplasmic signaling domains. Detection tags incorporated into the extracellular spacer provide a valuable identification marker for receptor surface expression. We observed dramatic differences in secretion and detection of surface expression levels for the analyzed constructs (Figure 1). Interestingly, both the $\mathrm{N}$ - or C-terminal incorporation of a Myc sequence completely impaired the secretion of the HEL-specific scFv, suggesting disturbed protein folding or secretion, which is more likely for the tag fused $\mathrm{N}$-terminally, as the tag sequence directly behind the signal peptide can interfere with translocation into the secretory pathway. Constructs containing a Strep tag II showed drastically improved detection and selection of cells engineered with a CBCR compared to constructs including a Flag tag sequence in the extracellular spacer region (Figures 2B, 3E and Figure S4). In contrast to the Myc containing constructs, the Flag tag still enabled surface expression, proposing compromised Flag tag accessibility. Extracellular linker sequences are expected to provide certain degrees of $\mathrm{scFv}$ flexibility, while still allowing signal transduction. Variations of linker length did not increase tag accessibility measured by tag detection and correlation with antigen binding (Figure 3). In a recent study, similar spacer regions including a Flag tag were used to successfully detect the surface expression of synthetic antigen receptors in HEK293FT cells, suggesting that detection and accessibility of an orthogonal tag sequence are additionally influenced by the cell type (43). Furthermore, the length and composition of extracellular spacers has been reported to be decisive for surface expression and activity of antigen receptors $(11,43)$. We tested a series of linker sequences, however did not observe any effect on surface expression (Figure 3). In contrast, we found that the transmembrane domain affected surface expression implying that the transmembrane region also has the capacity to provide stability to the CBCR (Figures 2, 3). CBCR encoding a CD28-derived transmembrane domain showed increased surface expression compared to the CBCR including an endogenous BCR-TM. This result is consistent with previous research revealing that the $\mathrm{CD} 28 \mathrm{TM}$ domain induces a higher expression of CAR than the CD3 $\zeta$ TM domain (55).

Our findings support that synthetic receptors require careful evaluation of their various components in order to have an optimized expression and detection system.

The polypeptides CD79 are required for the transport of a BCR to the cell surface and for signal transduction (56, 57). Our results show that the inferior surface expression of constructs containing the IgG2c-TM could be partially rescued by fusing the short intracellular tail to the cytoplasmic domain of a CD79 protein (Figures 4, 6). Previous work suggested that CD79 $\alpha$ and CD79 $\beta$ are independently sufficient to drive B cell maturation and activation, as long as the ITAM region of the intracellular signaling domain remain intact (47). In accordance with this previous work, our results reflect the improved surface transport of the CD79 $\beta$ construct compared to the CD79 $\alpha$ receptor (Figures 4, 6). We envision CBCR-engineered $\mathrm{B}$ cells equipped with the $C D 79 \beta$ signaling domain to, upon antigen exposure, undergo selective expansion and contribution to both the memory and plasma cell pool, not only providing long term supply of deficient protein from long-lived plasma cells, but also enabling the reactivation of engineered cells from the memory $\mathrm{B}$ cell pool by antigen re-exposure.

In order to evaluate highly expressed $\mathrm{CBCR}$ variants in primary $\mathrm{B}$ cells, we developed a reliable pipeline to genomically integrate large gene cassette by Cas9-driven HDR (Figure 5). While many years of work have aimed to reprogram immune cells for therapeutic purposes, such as CAR $\mathrm{T}$ cell therapy, these have almost exclusively relied on viral-based gene transfer. Recently, genome editing platforms providing targeted integration, most notably CRISPR-Cas9, have become promising tools to further improve current immune cell therapies, by offering potential advantages related to safety, uniform expression levels and potency $(54,58-60)$. Establishing a preclinical genome editing platform based on primary murine $\mathrm{B}$ cells does not only show progress on cellular engineering of technically challenging target cell lines, but also allows the investigation of these cells as novel vehicle for adoptive immune cell therapies. We observed robust transfection efficiencies (electroporation by nucleofection) in primary B cells following pre-activation and expansion on fibroblast feeder cells expressing BAFF and CD40 ligand. Cas9-RNP-mediated HDR of doublestranded DNA occurred consistently, but with relatively low efficiencies when compared to other primary lymphocyte cells, such as T cells $(49,59)$. Furthermore, in contrast to observations in multiple other cell types including $\mathrm{T}$ and stem cells, AAV 
A

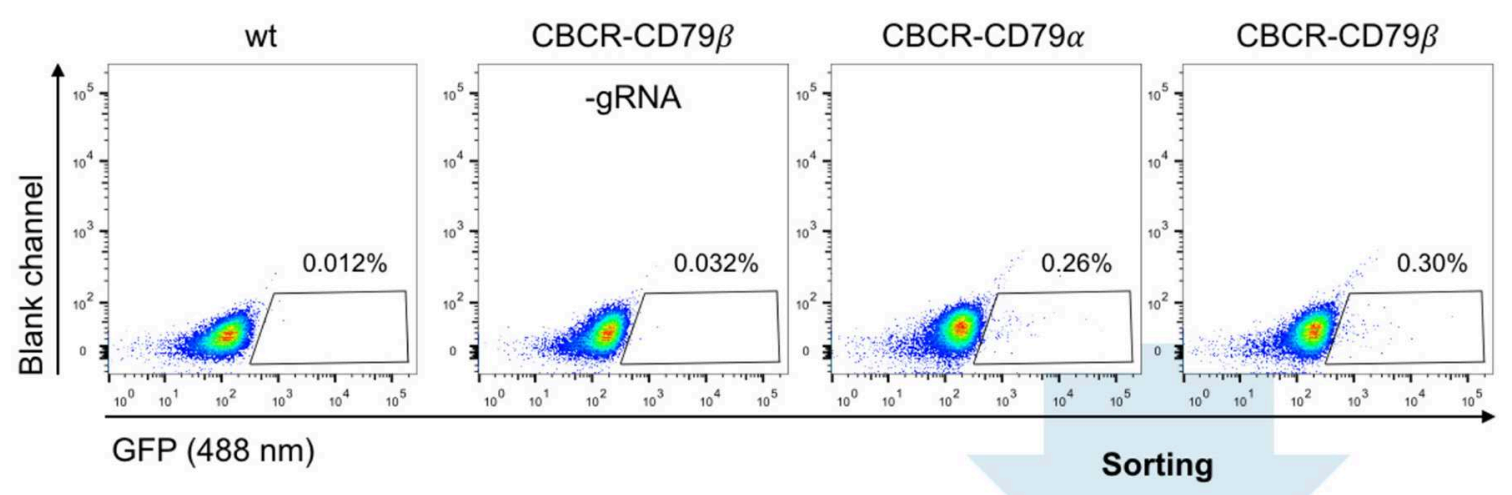

B

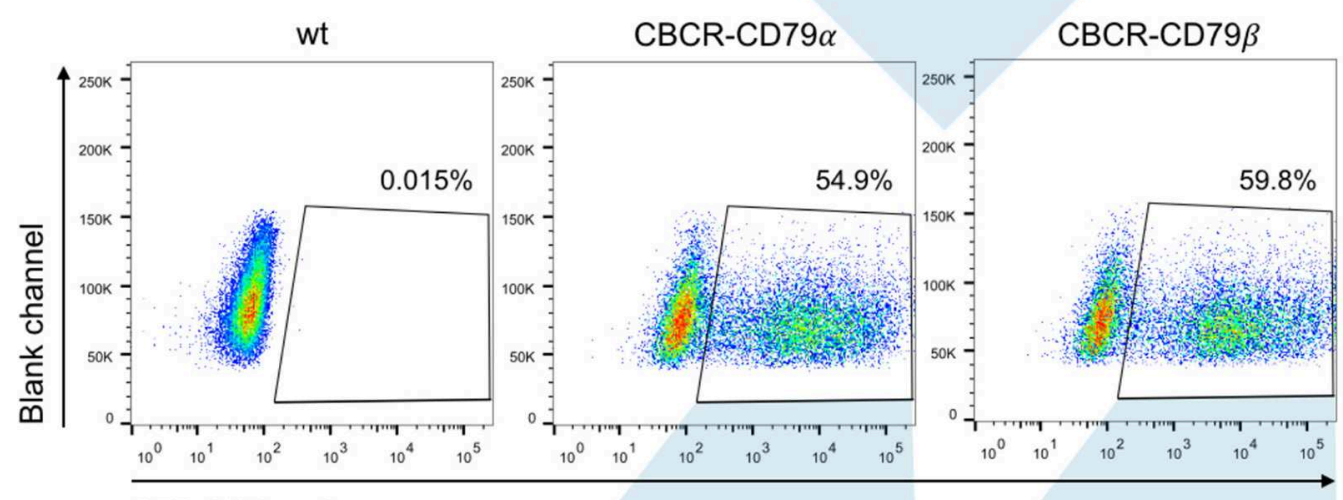

C
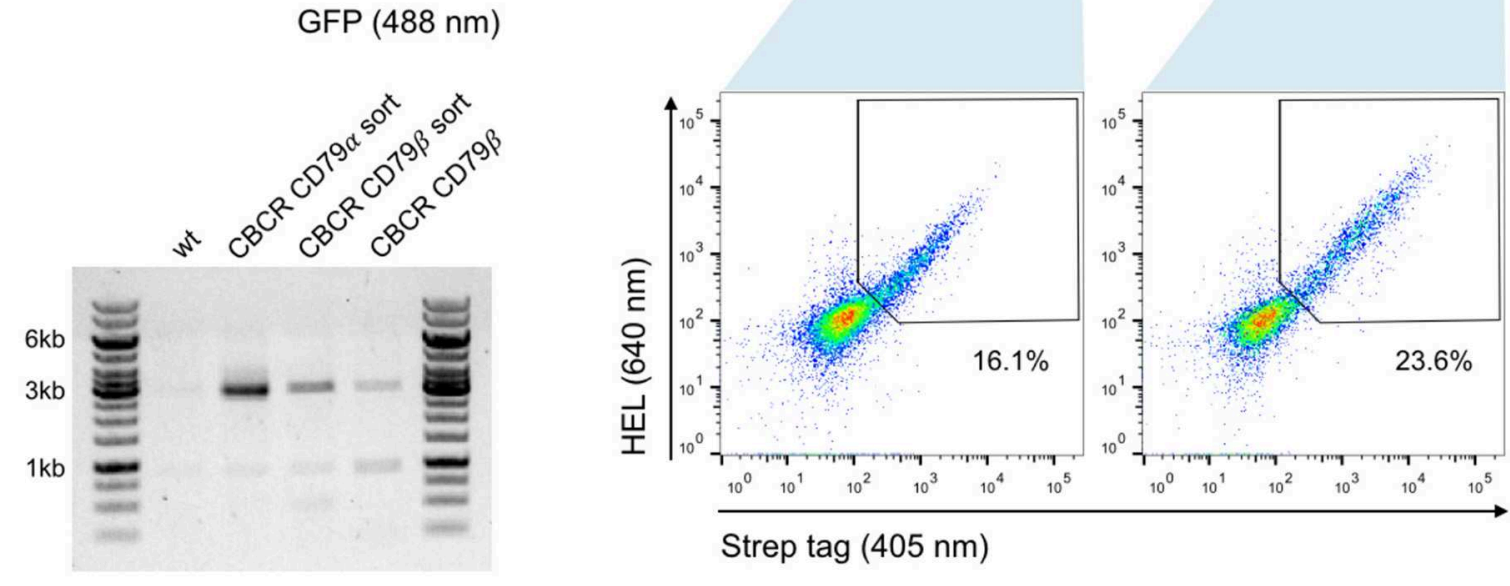

Strep tag (405 nm)

FIGURE 6 | Robust and stable CBCR surface expression in primary B cells. (A) Splenic B cells from C57BL/6-Ly5.1 mice were transfected with Cas9-RNP and HDR donor templates encoding synthetic CBCR (previously optimized in hybridoma cells, Figures $\mathbf{3}, 4$ ) following $24 \mathrm{~h}$ of pre-activation on a $40 \mathrm{LB}$ feeder cell layer and culture in the presence of IL-4. Integration efficiencies based on GFP expression ( $488 \mathrm{~nm})$ were determined by flow cytometry on day 3 after transfection and GFP+ cells were sorted. Primary B cells electroplated without gRNA and non-transfected B cells serve as negative controls. Flow cytometry plots are representative of three independent experiments. (B) Sorted primary B cells were successfully recovered during co-culture on 40 LB feeder cells and in the presence of IL-21. Flow cytometry dot plots show efficient enrichment of GFP ${ }^{+}$cells (upper row) and CBCR surface expression in primary B cells based on HEL antigen binding and detection of the Strep II tag within the GFP+ population (lower row). (C) Agarose gel shows genomic PCR products (p7/8) that confirm the targeted integration of the GFP-T2A-CBCR cassette containing the intracellular domain of either CD79 protein (3,052 bp) before and after sorting.

delivery of the HDR donor only marginally increased HDR frequencies in primary $B$ cells, suggesting that low HDR efficiencies are independent of template format and transfection efficiencies $(52,58)$. Notably, the AAV format caused a relatively high background of gene expression from episomal retention of DNA (Figures 5F,G). Our results imply that in constructs that use a constitutive promoter for gene expression, AAVbased template delivery in primary murine B cells may not be sensitive enough to effectively distinguish edited cells from episomal expression. It may be beneficial for approaches that are designed such that only correct integration leads to gene expression (i.e., splicing or use of endogenous promoter) (29, 
61). We found dramatically enhanced HDR-mediated integration efficiencies in hybridoma B cells using the same AAV-DJ template targeting the same genomic locus (Figure S3), thus the low HDR frequencies in primary murine B cells is not related generally to delivery or targeting of genome editing reagents. Although, using in vitro expansion methods (e.g., 40 LB feeder layers) (48) following genome editing of $\mathrm{B}$ cells may also be sufficient to overcome low HDR efficiencies, and enable functional testing of signaling and activation from synthetic receptors. In the future, it would be valuable to determine the potential causes for these inherent limitations of HDR, perhaps there is an upregulation of inhibitory factors for HDR in primary murine B cells. In this context, very low activity of conservative HDR known for its high-fidelity and predominant appearance of error-prone, nonconservative homologous recombination causing deletions, gene fusions and other genetic aberrations (62) has been described before. Although, in the context of human B cells, Cas9-RNP with AAV-6 donor has been reported to be highly efficient (at least $10 \%$; 100 -fold higher HDR rates than what we observed in this study), emphasizing once more that the differences between murine and human cells must not be underestimated $(19,34)$. Given previously described discrepancies in both innate and adaptive immunity, it is not surprising that differences between murine and human cells may also occur in DNA repair mechanisms, suggesting human B cells as promising vehicle for adoptive immune cell therapies.

The clear discrimination of edited cells using PCR-derived HDR donor still offers a very reliable tool to develop new concepts for cellular therapies. Recently, Hung et al. used an interesting strategy in primary human B cells by combining gene disruption for plasma cell differentiation with engineering of these cells to secrete a therapeutic protein, followed by in vivo transfer in immunodeficient mice (19). To further evaluate and optimize in vivo stability, our approach for cellular engineering in primary murine $\mathrm{B}$ cells enables studies that perform adoptive transfer to immunocompetent mouse models, which will be valuable for developing novel B cell-based immunotherapies. Additionally, an alternative therapeutic application is to utilize CBCRs to target cytokines such as tumor necrosis factoralpha (TNF-a) or IL-6, which are over-expressed in chronic inflammatory diseases (e.g., rheumatoid arthritis). Finally, recent work has demonstrated the integration of synthetic antibodies into the endogenous BCR locus suggesting the potential of $\mathrm{B}$ cell therapies for protection from chronic viral infections such as HIV-1 or RSV (63-65). Directly engineering the humoral immune response to enable the durable and potent generation of neutralizing antibodies represents a possible strategy to overcome the current challenges in traditional vaccination.

\section{METHODS}

\section{Preparation of HDR Donor Templates}

All primers were ordered from Integrated DNA Technologies (IDT) and sequences are listed in Table S1. HDR donor constructs were cloned by Gibson assembly using the Gibson Assembly Master Mix (NEB, E2611S) into the pUC57(Kan) cloning vector, obtained from Genewiz. The vector was designed with homology arms PCR-amplified from C57BL/6Ly5.1 genomic DNA according to the mouse genomic sequence [Gt(ROSA)26Sor gene] and Sanger sequenced (pUC57-Rosa26). Codon-optimized nucleotide sequences encoding each transgene or parts of it were synthesized (gBlocks, IDT DNA Technologies) or generated by PCR from previously characterized CBCR expression vectors. Anti-HEL scFv was derived from the high-affinity HyHEL10 antibody in the $\mathrm{V}_{\mathrm{L}}-\mathrm{V}_{\mathrm{H}}$ format or codon-optimized for mice from the D1.3 variant M3 scFv (CA2787677A1). M3 scFv was linked by extracellular spacer regions incorporating different detection tags (Myc, Flag, Strep II) to either a BCR or CD28 transmembrane domain. These TM domains are fused to the short intracellular tail of the BCR Cterminally followed by the cytosolic domain of either the CD79 $\alpha$ or CD79 $\beta$ polyprotein. All CBCRs contain an N-terminal $V_{\kappa}$ signal peptide for membrane targeting. The GFP reporter gene and T2A-CBCR constructs were cloned into the pUC57-Rosa26 under the control of a CMV promoter. HDR donor vectors were linearized by PCR with the KAPA Hifi HotStart ReadyMix (KAPA Biosystems, KK2602) using either p 9 and p10 or p11 and p12 (HA 750 bp each) for direct comparison with AAV delivered HDR donor. For each PCR, the reaction was split between a minimum of five separate tubes and then pooled for subsequent steps. This split-pool PCR approach was used to minimize the chance of mutations in the repair template arising from PCR. The PCR product was purified using DNA Clean \& Concentrator ${ }^{\mathrm{TM}}-5$ (Zymo, D4013), eluted in ddH2O, and concentrated to $\sim 1 \mu \mathrm{g} / \mu \mathrm{l}$ using a Concentrator 5,301 (Eppendorf).

\section{Mice}

C57BL/6-Ly5.1 mice were obtained by in house breeding and were maintained in the mouse facility under specific pathogen-free conditions. Mouse procedures were performed under protocols approved by the Basel-Stadt cantonal veterinary office (Basel-Stadt Kantonales Veterinäramt Tierversuchsbewilligung \#2701).

\section{Cell Culture}

All cell lines were maintained in incubators at $37^{\circ} \mathrm{C}$ and $5 \% \mathrm{CO}_{2}$ and were confirmed to be negative for Mycoplasma contamination using the (ATCC, 30-1012K). If required, the live cell number was counted by the trypan blue dye exclusion method using the TC 10 Automated Cell Counter (Bio-Rad). All B cell hybridoma lines were cultivated in high-glucose Dulbecco's Modified Eagle Medium (DMEM) containing GlutaMAX supplemented with $10 \% \mathrm{FBS}, 100 \mathrm{U} \mathrm{ml}^{-1}$ penicillin/streptomycin, $10 \mathrm{mM}$ HEPES buffer and $50 \mu \mathrm{M} 2$ mercaptoethanol. Hybridomas were typically maintained as $5 \mathrm{~mL}$ cultures in T-25 flasks and passaged every $48-72 \mathrm{~h}$. A list of all hybridoma cell lines is provided in Table S3. Balb/c 3T3 fibroblast derived $40 \mathrm{LB}$ feeder cells were previously generated by Nojima et al. (48), maintained in high-glucose DMEM containing GlutaMAX supplemented with $10 \%$ FBS and $100 \mathrm{U} \mathrm{ml}^{-1}$ and passaged at $90 \%$ confluence. To prepare the feeder layer, $40 \mathrm{LB}$ cells were plated at $4 \times 10^{4}$ per $\mathrm{cm}^{2}$ about $16 \mathrm{~h}$ before coculture and irradiated with 60 Gy $\gamma$-ray. Splenic B cells were pre-activated in a T-75 flask in the presence of irradiated 40 
LB feeder cells in $40 \mathrm{~mL}$ RPMI-1630 medium supplemented with $10 \%$ FBS of a particular lot number, $1 \mathrm{mM}$ Na-Pyruvate, $10 \mathrm{mM}$ HEPES buffer, $100 \mathrm{U} \mathrm{ml}^{-1}$ Penicillin/Streptomycin and $50 \mu \mathrm{M}$ M2-mercaptoethanol for 24h. rIL-4 (1 ng ml $\mathrm{m}^{-1}$, Peprotech) was added to the primary culture for 4 days. From day 4 , cells were cultivated on a new feeder layer with rIL-21 (10 ng ml ${ }^{-1}$, Peprotech).

\section{Splenic B Cell Isolation}

Single cell suspension of splenocytes were generated from C57BL/6-Ly5.1 mouse spleen under sterile conditions by passing cells through a $70 \mu \mathrm{M}$ cell strainer using the plunge of a syringe. Subsequently, cells were counted and pelleted at $300 \mathrm{~g}$ for $10 \mathrm{~min}$ at $4{ }^{\circ} \mathrm{C}$ before resuspending them in autoMACS running buffer (Miltenyi Biotech). Highly pure resting B cells were isolated by magnetic labeling and depletion of CD43-expressing B cells and non-B cells using the Mouse B cell Isolation Kit (Miltenyi Biotech, 130-090-862) and MACS LS columns (Miltenyi Biotech) following vendor instructions. For activation, up to $3 \times 10^{7}$ cells were plated in a T-75 flask on a $40 \mathrm{LB}$ feeder cell layer for $24 \mathrm{~h}$, if not described differently.

\section{Gene Editing in Primary Murine B Cells}

Twenty-Four hours after initiating $B$ cell activation on the feeder layer, B cells including the $40 \mathrm{LB}$ feeder cells were harvested by collecting the growth medium and dissociating the adherent cells by adding $3 \mathrm{~mL}$ autoMACS running buffer (Miltenyi Biotech) to the T-75 flask. Prior to transfection, customized Alt-R crRNA and Alt-R tracrRNA (Alt-R ${ }^{\circledR}$ CRISPRCas9 System, IDT) were complexed in equimolar concentrations by incubation at $95^{\circ} \mathrm{C}$ for $5 \mathrm{~min}$. crRNAs were designed using the Broad institute single guide RNA (sgRNA) design tool (http://portals.broadinstitute.org/gpp/public/analysis-tools/ sgrna-design). Sequences of all tested gRNAs are listed in Table S2. All genome editing experiments performed utilized Cas9 from Streptococcus pyogenes (SpCas9) purchased from IDT. Pre-activated $\mathrm{B}$ cells were transfected using the P4 Primary Cell 4D-Nucleofector X Kit L (Lonza, V4XP-4024) in combination with the program DI-100. The following standard conditions in $100 \mu \mathrm{l}$ total volume of nucleofection mix were used, if not described differently: $1 \times 10^{6}$ cells, $20 \mu \mathrm{g}$ Cas 9 protein complexed with $0.156 \mathrm{nmol}$ Alt-R duplex gRNA at 1:1.125 ratio and $5 \mu \mathrm{g}$ of linearized double-stranded DNA generated by PCR. After electroporation, edited cells were seeded in 5-6 mL culturing medium supplemented with rIL-4 into a 6-well plate in the presence of irradiated $40 \mathrm{LB}$ cells $\left(5 \times 10^{5}\right.$ cells per well $)$. Two days after transfection (Day 3 ), the $\mathrm{B}$ cell culturing medium was replaced by carefully aspirating the medium and adding $5 \mathrm{ml}$ of fresh B cell medium supplemented with rIL-4. One day later (Day 4), primary B cells were harvested and prepared for flow cytometry analysis.

\section{Gene Editing in Hybridoma Cells}

Genome editing experiments in B cell hybridomas were always executed in the HC9- cell line being dysfunctional in antibody expression and constitutively expressing Cas9 protein (30).
Hybridoma cells were electroporated using the SF Cell Line 4DNucleofector X Kit L (Lonza, V4XC-2024) with the program CQ-104. The following standard conditions in $100 \mu \mathrm{l}$ of total volume of nucleofection mix were used: $1 \times 10^{6}$ cells, 0.156 nmol pre-complexed Alt-R duplex gRNA and $5 \mu \mathrm{g}$ of PCRlinearized double-stranded DNA. Following transfection, cells were incubated for $5 \mathrm{~min}$ at RT, before adding $500 \mu \mathrm{l}$ of prewarmed medium to the nucleocuvette and typically transferring them to $1.5 \mathrm{~mL}$ of fresh growth medium in 6-well plates. The cells were usually supplemented $24 \mathrm{~h}$ later with $0.5-1.0 \mathrm{~mL}$ of fresh culturing medium.

\section{Transduction With AAV}

AAV vector plasmids were cloned in the pMD13-AAV plasmid containing inverted terminal repeats from AAV serotype 2 . HDR donor cassettes including a GFP gene under the control of a CMV promoter and a SV40 polyA sequence flanked by 750 bp homology arms of the Rosa26 locus were inserted into the MCS by NotI restriction enzyme. Cloning was performed in the suitable bacterial strain Stbl3. AAV stocks were produced by the Viral Vector Facility (VVF) of the Neuroscience Center Zurich. The optimal AAV serotype for the transduction of primary murine B cells was evaluated by adding ssAAV with a GFP coding sequence packaged using various serotypes (AAV1, 2, 6, 7, 8, 9, DJ) at an MOI of $2.5 \times 10^{10}$ to B cells pre-activated for $24 \mathrm{~h}$. For samples transduced with AAV for HDR template delivery, AAV-DJ donor vector was added to the culture immediately after electroporation at an MOI (vector genomes/cell) of 20,000$5 \times 10^{5}$ and cultured as described for transfected cells. AAV donor was added as $10 \%$ of the final culture volume regardless of titer.

\section{Genomic Analysis of CRISPR-Cas9 Targeting}

The activity of gRNAs targeting the Rosa26 locus were initially tested by induction of NHEJ. Cells transfected with Cas9RNP targeting the Rosa26 locus were harvested 4 days after electroporation, washed once in PBS and genomic DNA was recovered from $1 \times 10^{6}$ cells using $100 \mu$ l Quick Extract solution (Epicenter) according to the manufacturer's instructions. Small fragments of DNA covering the putative cleavage sites were amplified by PCR with KAPA Hifi HotStart Ready Mix (KAPA Biosystems, KK2602) from the genomic DNA using primers p13 and p14. Control DNA was also amplified from wildtype C57BL/6-Ly5.1 genomic DNA. CRISPR-Cas9 cleavage of the genome was determined using a Surveyor Mutation Detection Kit (IDT, 706020). All samples were run on $2 \%$ gels for the detection of cleavage products. For reference, GeneRuler $1 \mathrm{~kb}$ DNA Ladder (Thermo, SM0314) and GeneRuler 100 bp DNA Ladder (Thermo, SM0243) were used as DNA size markers.

\section{Measuring Targeted Integration of CBCR Construct}

For the evaluation of transgene integration, PCR analysis was performed on genomic DNA extracted from sorted cells or single-cell clones excluding the presence of remaining repair template. Primer p3 and p4, closely flanking the gRNA targeting 
site in the Rosa26 locus in combination with KAPA Hifi HotStart Ready Mix were used with the following PCR conditions: 35 cycles with annealing at $62^{\circ} \mathrm{C}(15 \mathrm{~s})$, elongation at $72^{\circ} \mathrm{C}$ (1:30 $\mathrm{min})$ and final elongation at $72^{\circ} \mathrm{C}(3: 00 \mathrm{~min})$.

To determine targeted integration mediated via HDR, PCR was performed on genomic DNA using primers binding inside the construct cassette and outside of homology arm. Primer p5 and p6 were used with the following cycling conditions: 35 cycles with annealing at $69{ }^{\circ} \mathrm{C}(15 \mathrm{~s})$, elongation at $72^{\circ} \mathrm{C}(1: 30 \mathrm{~min})$, final elongation at $72^{\circ} \mathrm{C}(3: 00 \mathrm{~min})$, primer $\mathrm{p} 7$ and p8: 35 cycles with annealing at $71^{\circ} \mathrm{C}(15 \mathrm{~s})$, elongation at $72^{\circ} \mathrm{C}(1: 30 \mathrm{~min})$, final elongation at $72^{\circ} \mathrm{C}(3: 00 \mathrm{~min})$, primer $\mathrm{p} 7$ and $\mathrm{p} 15: 35$ cycles with annealing at $73^{\circ} \mathrm{C}(15 \mathrm{~s})$, elongation at $72^{\circ} \mathrm{C}(1: 30 \mathrm{~min})$, final elongation at $72^{\circ} \mathrm{C}(3: 00 \mathrm{~min})$.

\section{Evaluation of scFv Expression by RT-PCR}

To confirm transcript expression of the HEL-specific scFv variants, mRNA was isolated from $1 \times 10^{6}$ transfected or GFPbulk sorted hybridoma and parental HC9- cells using $200 \mu \mathrm{l}$ TRIzol ${ }^{\circledR}$ reagent (Thermo, 15596-026). The mRNA was purified using the PureLink Mini Kit (Invitrogen, Thermo) according to the manufacturer's instructions. First-strand cDNA was synthesized from mRNA using Maxima Reverse Transcriptase (Thermo, EP0742) and used as template DNA for subsequent PCR reactions. For the detection of correct transcript expression, the following cycling conditions were applied using KAPA Hifi HotStart Ready Mix and p1 and p2, binding to GFP and the SV40 polyA sequence: 25 cycles with annealing at $61^{\circ} \mathrm{C}(15 \mathrm{~s})$, elongation at $72^{\circ} \mathrm{C}(1 \mathrm{~min})$, final elongation at $72^{\circ} \mathrm{C}(2 \mathrm{~min})$.

\section{Measuring scFv Secretion by ELISA}

Three days prior to measuring culture scFv levels, GFP+ sorted cells were collected, counted and then resuspended in new culture medium. After 3 days, the cell culture supernatant was collected from $1 \times 10^{6}$ cells and normalized to leastconcentrated sample. scFv secretion levels were analyzed by ELISA after coating with HEL antigen (Sigma-Aldrich, 62971, $4 \mu \mathrm{g} \mathrm{ml}-1$ ) in PBS (Thermo, 10010-015). The plates were then blocked with PBS supplemented with $2 \% \mathrm{~m} / \mathrm{v}$ milk (AppliChem, A0830) and 0.05\% V/V Tween-20 (AppliChem, A1389, PBSMT) followed by three washing steps with PBS supplemented with Tween-20 0.05\% V/V (PBST). Supernatants were then serially diluted (at 1:3 ratio) in PBSTM, starting from the non-diluted supernatant as the highest concentration. Supernatants were incubated for $1 \mathrm{~h}$ at RT, followed by three washing steps with PBST and incubation with HRPconjugated anti-Myc antibody (9E10, Thermo Fisher Scientific, MA1-81357) or anti-Flag antibody (FG4R, Thermo Fisher Scientific, MA1-91878-HRP) at $2 \mu \mathrm{g} \mathrm{mL}-1$ (1:500 dilution from stock) in PBSTM. After three more washing steps with PBST, ELISA detection was performed using a 1Step Ultra TMB-ELISA Substrate Solution (Thermo, 34028), reaction was terminated with $\mathrm{H}_{2} \mathrm{SO}_{4}(1 \mathrm{M})$. Antibodies and working concentrations are described in Table S4. Absorbance at $450 \mathrm{~nm}$ was measured using an Infinite 200PRO NanoQuant reader (Tecan). ELISA data were analyzed with the software GraphPad Prism.

\section{Flow Cytometry Analysis and Sorting for Immunophenotyping}

Flow cytometry-based analysis and cell isolation were performed on a 5 laser BD LSR Fortessa ${ }^{\mathrm{TM}}$ flow cytometer and BD FACS Aria III (BD Biosciences), respectively. Data were analyzed with FlowJo software (Tree Star).

Twenty-four hours post transfection in any of the cell lines, $\sim 100 \mu \mathrm{l}$ were collected and analyzed for GFP expression (via GFP-T2A). Primary B cells were only harvested for sorting on GFP expression 3 days after transfection. Hybridoma cells were enriched for GFP expressing cells 3 days post transfection, if not indicated differently. After sorting and expansion primary B cells or hybridoma cells were labeled with HEL-antigen, conjugated to Alexa Fluor 647 dye using the Alexa Fluor ${ }^{\circledR} 647$ Protein Labeling Kit (Thermo Fisher Scientific, A20173) according to the manufacturer's instructions, and antibodies binding the respective detection tag to determine CBCR surface expression. For this purpose, cells were washed with phosphate-buffered saline (PBS), incubated with the labeling antibody or antigen for $30 \mathrm{~min}$ on ice or $10 \mathrm{~min}$ at RT, protected from light, washed again with PBS and analyzed or sorted. Biotinylated antibodies were stained with StrepatvidinBV421 (Biolegend). Staining with propidium iodide (PI, BD BioSciences) was used for live/dead cell discrimination as directed by the manufacturer. When primary B cells that had been cultured on a $40 \mathrm{LB}$ feeder layer were analyzed, 40 LB feeder cells were excluded based on FSC vs. SSC. The labeling reagents and working concentrations are described in Table S5.

\section{DATA AVAILABILITY STATEMENT}

All datasets generated for this study are included in the article/Supplementary Material.

\section{ETHICS STATEMENT}

The animal study was reviewed and approved by Basel-Stadt Cantonal Veterinary Authority.

\section{AUTHOR CONTRIBUTIONS}

TP and SR developed methodology and designed experiments. TP and LB performed experiments. WK, CP, RE, LC, and DK generated critical materials and provided technical advice. TP, LB, and SR analyzed data and wrote manuscript.

\section{FUNDING}

Funding was provided by the National Competence Center for Research on Molecular Systems Engineering.

\section{ACKNOWLEDGMENTS}

We acknowledge the ETH Zurich D-BSSE Single Cell Unit and the ETH Zurich D-BSSE Animal Facility for support, 
in particular, T. Lopes, V. Jäggin, Marie-Didiée-Hussherr, and Gieri Camenisch. We are grateful to Mark Pogson for providing initial scientific discussions and feedback. This manuscript has been released as a Pre-print at bioRxiv (66).

\section{REFERENCES}

1. Kalos M, Levine BL, Porter DL, Katz S, Grupp SA, Bagg A, et al. T cells with chimeric antigen receptors have potent antitumor effects and can establish memory in patients with advanced leukemia. Sci Transl Med. (2011) 3:95ra73. doi: 10.1126/scitranslmed.3002842

2. Ronson A, Tvito A, Rowe JM. Treatment of relapsed/refractory acute lymphoblastic leukemia in adults. Curr Oncol Rep. (2016) 18:39. doi: $10.1007 /$ s11912-016-0519-8

3. Jain MD, Bachmeier CA, Phuoc VH, Chavez JC. Axicabtagene ciloleucel (KTE-C19), an anti-CD19 CAR T therapy for the treatment of relapsed/refractory aggressive B-cell non-Hodgkin's lymphoma. Ther Clin Risk Manage. (2018) 14:1007-17. doi: 10.2147/TCRM. S145039

4. Friedman KM, Garrett TE, Evans JW, Horton HM, Latimer HJ, Seidel $\mathrm{SL}$, et al. Effective targeting of multiple B-cell maturation antigenexpressing hematological malignances by anti-B-cell maturation antigen chimeric antigen receptor T cells. Hum Gene Ther. (2018) 29:585-601. doi: 10.1089/hum.2018.001

5. Zhang N, Bevan MJ. CD8+ T cells: foot soldiers of the immune system. Immunity. (2011) 35:161-8. doi: 10.1016/j.immuni.2011.07.010

6. Lim WA, June $\mathrm{CH}$. The principles of engineering immune cells to treat cancer. Cell. (2017) 168:724-40. doi: 10.1016/j.cell.2017.01.016

7. Turtle CJ, Hanafi L-A, Berger C, Gooley TA, Cherian S, Hudecek M, et al. CD19 CAR-T cells of defined CD4+:CD8+ composition in adult B cell ALL patients. J Clin Invest. (2016) 126:2123-38. doi: 10.1172/JCI85309

8. Maude SL, Barrett D, Teachey DT, Grupp SA. Managing cytokine release syndrome associated with novel T cell-engaging therapies. Cancer J Sudbury Mass. (2014) 20:119-22. doi: 10.1097/PPO.0000000000000035

9. Kuwana Y, Asakura Y, Utsunomiya N, Nakanishi M, Arata Y, Itoh S, et al. Expression of chimeric receptor composed of immunoglobulin-derived V resions and T-cell receptor-derived C regions. Biochem Biophys Res Commun. (1987) 149:960-8. doi: 10.1016/0006-291X(87)90502-X

10. June CH, O'Connor RS, Kawalekar OU, Ghassemi S, Milone MC. CAR $\mathrm{T}$ cell immunotherapy for human cancer. Science. (2018) 359:1361-5. doi: 10.1126/science.aar6711

11. Hudecek M, Sommermeyer D, Kosasih PL, Silva-Benedict A, Liu L, Rader $\mathrm{C}$, et al. The non-signaling extracellular spacer domain of chimeric antigen receptors is decisive for in vivo antitumor activity. Cancer Immunol Res. (2015) 3:125-35. doi: 10.1158/2326-6066.CIR-14-0127

12. Kudo K, Imai C, Lorenzini P, Kamiya $T$, Kono K, Davidoff AM, et al. $\mathrm{T}$ lymphocytes expressing a CD16 signaling receptor exert antibody-dependent cancer cell killing. Cancer Res. (2014) 74:93-103. doi: 10.1158/0008-5472.CAN-13-1365

13. De Munter S, Ingels J, Goetgeluk G, Bonte S, Pille $M$, Weening $K$, et al. Nanobody based dual specific CARs. Int J Mol Sci. (2018) 19:403. doi: 10.3390/ijms19020403

14. Urbanska K, Lanitis E, Poussin M, Lynn R, Gavin BP, Kelderman S, et al. A universal strategy for adoptive immunotherapy of cancer through use of a novel T cell antigen receptor. Cancer Res. (2012) 72:1844-52. doi: 10.1158/0008-5472.CAN-11-3890

15. Guedan S, Posey AD, Shaw C, Wing A, Da T, Patel PR, et al. Enhancing CAR T cell persistence through ICOS and 4-1BB costimulation. JCI Insight. (2018) 3:96976. doi: 10.1172/jci.insight.96976

16. Ali SA, Shi V, Maric I, Wang M, Stroncek DF, Rose JJ, et al. T cells expressing an anti-B-cell maturation antigen chimeric antigen receptor cause remissions of multiple myeloma. Blood. (2016) 128:1688-700. doi: 10.1182/blood-2016-04-711903

\section{SUPPLEMENTARY MATERIAL}

The Supplementary Material for this article can be found online at: https://www.frontiersin.org/articles/10.3389/fimmu. 2019.02630/full\#supplementary-material

17. Slifka MK, Antia R, Whitmire JK, Ahmed R. Humoral immunity due to long-lived plasma cells. Immunity. (1998) 8:363-72. doi: 10.1016/S1074-7613(00)80541-5

18. Radbruch A, Muehlinghaus G, Luger EO, Inamine A, Smith KGC, Dörner T, et al. Competence and competition: the challenge of becoming a long-lived plasma cell. Nat Rev Immunol. (2006) 6:741-50. doi: 10.1038/nri1886

19. Hung KL, Meitlis I, Hale M, Chen C-Y, Singh S, Jackson SW, et al. Engineering protein-secreting plasma cells by homology-directed repair in primary human B cells. Mol Ther. (2018) 26:456-67. doi: 10.1016/j.ymthe.2017.11.012

20. Kim E-K, Seo H-S, Chae M-J, Jeon I-S, Song B-Y, Park Y-J, et al. Enhanced antitumor immunotherapeutic effect of B-cell-based vaccine transduced with modified adenoviral vector containing type 35 fiber structures. Gene Ther. (2014) 21:106-14. doi: 10.1038/gt.2013.65

21. Hellebrand E, Mautner J, Reisbach G, Nimmerjahn F, Hallek M, Mocikat $\mathrm{R}$, et al. Epstein-Barr virus vector-mediated gene transfer into human B cells: potential for antitumor vaccination. Gene Ther. (2006) 13:150-62. doi: $10.1038 /$ sj.gt.3302602

22. Serafini M, Naldini L, Introna M. Molecular evidence of inefficient transduction of proliferating human B lymphocytes by VSVpseudotyped HIV-1-derived lentivectors. Virology. (2004) 325:413-24. doi: 10.1016/j.virol.2004.04.038

23. Frecha C, Costa C, Levy C, Negre D, Russell SJ, Maisner A, et al. Efficient and stable transduction of resting B lymphocytes and primary chronic lymphocyte leukemia cells using measles virus gp displaying lentiviral vectors. Blood. (2009) 114:3173-80. doi: 10.1182/blood-2009-05-220798

24. Calderón-Gómez E, Lampropoulou V, Shen P, Neves P, Roch T, Stervbo U, et al. Reprogrammed quiescent B cells provide an effective cellular therapy against chronic experimental autoimmune encephalomyelitis. Eur J Immunol. (2011) 41:1696-708. doi: 10.1002/eji.201041041

25. Wang X, Moghimi B, Zolotukhin I, Morel LM, Cao O, Herzog RW. Immune tolerance induction to factor IX through B cell gene transfer: TLR9 signaling delineates between tolerogenic and immunogenic B cells. Mol Ther. (2014) 22:1139-50. doi: 10.1038/mt.2014.43

26. Cong L, Ran FA, Cox D, Lin S, Barretto R, Habib N, et al. Multiplex genome engineering using CRISPR/Cas systems. Science. (2013) 339:819-23. doi: 10.1126/science. 1231143

27. Mali P, Yang L, Esvelt KM, Aach J, Guell M, DiCarlo JE, et al. RNAguided human genome engineering via Cas9. Science. (2013) 339:823-6. doi: 10.1126/science.1232033

28. Hsu PD, Lander ES, Zhang F. Development and applications of CRISPR-Cas9 for genome engineering. Cell. (2014) 157:1262-78. doi: 10.1016/j.cell.2014.05.010

29. Pogson M, Parola C, Kelton WJ, Heuberger P, Reddy ST. Immunogenomic engineering of a plug-and-(dis)play hybridoma platform. Nat Commun. (2016) 7:12535. doi: 10.1038/ncomms12535

30. Mason DM, Weber CR, Parola C, Meng SM, Greiff V, Kelton WJ, et al. High-throughput antibody engineering in mammalian cells by CRISPR/Cas9mediated homology-directed mutagenesis. Nucleic Acids Res. (2018) 46:743649. doi: 10.1093/nar/gky550

31. Cheong T-C, Compagno M, Chiarle R. Editing of mouse and human immunoglobulin genes by CRISPR-Cas9 system. Nat Commun. (2016) 7:10934. doi: 10.1038/ncomms10934

32. Chu VT, Graf R, Wirtz T, Weber T, Favret J, Li X, et al. Efficient CRISPRmediated mutagenesis in primary immune cells using CrispRGold and a C57BL/6 Cas9 transgenic mouse line. Proc Natl Acad Sci USA. (2016) 113:12514-9. doi: 10.1073/pnas.1613884113

33. Wu C-AM, Roth TL, Baglaenko Y, Ferri DM, Brauer P, ZunigaPflucker JC, et al. Genetic engineering in primary human B cells with 
CRISPR-Cas9 ribonucleoproteins. J Immunol Methods. (2018) 457:33-40. doi: 10.1016/j.jim.2018.03.009

34. Johnson MJ, Laoharawee K, Lahr WS, Webber BR, Moriarity BS. Engineering of primary human B cells with CRISPR/Cas9 targeted nuclease. Sci Rep. (2018) 8:12144. doi: 10.1038/s41598-018-30358-0

35. Roybal KT, Williams JZ, Morsut L, Rupp LJ, Kolinko I, Choe JH, et al. Engineering $\mathrm{T}$ cells with customized therapeutic response programs using synthetic notch receptors. Cell. (2016) 167:419-32.e16. doi: 10.1016/j.cell.2016.09.011

36. Wörn A, Plückthun A. Stability engineering of antibody single-chain Fv fragments. J Mol Biol. (2001) 305:989-1010. doi: 10.1006/jmbi.2000.4265

37. Phan TG, Amesbury M, Gardam S, Crosbie J, Hasbold J, Hodgkin PD, et al. B cell receptor-independent stimuli trigger immunoglobulin (Ig) class switch recombination and production of IgG autoantibodies by anergic self-reactive B cells. J Exp Med. (2003) 197:845-60. doi: 10.1084/jem.20022144

38. Smith-Gill SJ, Wilson AC, Potter M, Prager EM, Feldmann RJ, Mainhart CR. Mapping the antigenic epitope for a monoclonal antibody against lysozyme. $J$ Immunol Baltim Md 1950. (1982) 128:314-22.

39. Chothia C, Lesk AM, Levitt M, Amit AG, Mariuzza RA, Phillips SE, et al. The predicted structure of immunoglobulin D1.3 and its comparison with the crystal structure. Science. (1986) 233:755-8. doi: 10.1126/science.3090684

40. Hawkins RE, Russell SJ, Baier M, Winter G. The contribution of contact and non-contact residues of antibody in the affinity of binding to antigen: the interaction of mutant D1.3 antibodies with lysozyme. J Mol Biol. (1993) 234:958-64. doi: 10.1006/jmbi.1993.1650

41. Kieback E, Charo J, Sommermeyer D, Blankenstein T, Uckert W. A safeguard eliminates $\mathrm{T}$ cell receptor gene-modified autoreactive $\mathrm{T}$ cells after adoptive transfer. Proc Natl Acad Sci USA. (2008) 105:623-8. doi: 10.1073/pnas.0710198105

42. Berahovich R, Xu S, Zhou H, Harto H, Xu Q, Garcia A, et al. FLAG-tagged CD19-specific CAR-T cells eliminate CD19-bearing solid tumor cells in vitro and in vivo. Front Biosci Landmark Ed. (2017) 22:1644-54. doi: 10.2741/4563

43. Schwarz KA, Daringer NM, Dolberg TB, Leonard JN. Rewiring human cellular input-output using modular extracellular sensors. Nat Chem Biol. (2017) 13:202-9. doi: 10.1038/nchembio.2253

44. Chang ZL, Chen YY. CARs: synthetic immunoreceptors for cancer therapy and beyond. Trends Mol Med. (2017) 23:430-50. doi: 10.1016/j.molmed.2017.03.002

45. Wang LD, Clark MR. B-cell antigen-receptor signalling in lymphocyte development. Immunology. (2003) 110:411-20. doi: $10.1111 / j .1365-2567.2003 .01756 . x$

46. Isakov N. Role of immunoreceptor tyrosine-based activation motif in signal transduction from antigen and Fc receptors. Adv Immunol. (1998) 69:183247. doi: 10.1016/S0065-2776(08)60608-2

47. Teh Y-M, Neuberger MS. The immunoglobulin (Ig) $\alpha$ and Ig $\beta$ cytoplasmic domains are independently sufficient to signal B cell maturation and activation in transgenic mice. J Exp Med. (1997) 185:1753-8. doi: $10.1084 /$ jem.185.10.1753

48. Nojima T, Haniuda K, Moutai T, Matsudaira M, Mizokawa S, Shiratori I, et al. In-vitro derived germinal centre B cells differentially generate memory B or plasma cells in vivo. Nat Commun. (2011) 2:465. doi: 10.1038/ ncomms 1475

49. Kornete M, Marone R, Jeker LT. Highly efficient and versatile plasmidbased gene editing in primary T cells. J Immunol. (2018) 200:2489-501. doi: 10.4049/jimmunol.1701121

50. Bialk P, Rivera-Torres N, Strouse B, Kmiec EB. Regulation of gene editing activity directed by single-stranded oligonucleotides and CRISPR/Cas9 systems. PLoS ONE. (2015) 10:e0129308. doi: 10.1371/journal.pone. 0129308

51. Richardson CD, Ray GJ, DeWitt MA, Curie GL, Corn JE. Enhancing homology-directed genome editing by catalytically active and inactive CRISPR-Cas9 using asymmetric donor DNA. Nat Biotechnol. (2016) 34:339. doi: $10.1038 /$ nbt. 3481
52. Dever DP, Bak RO, Reinisch A, Camarena J, Washington G, Nicolas CE, et al. CRISPR/Cas9 $\beta$-globin gene targeting in human haematopoietic stem cells. Nature. (2016) 539:384. doi: 10.1038/nature20134

53. Gaj T, Staahl BT, Rodrigues GMC, Limsirichai P, Ekman FK, Doudna JA, Schaffer DV. Targeted gene knock-in by homology-directed genome editing using Cas9 ribonucleoprotein and AAV donor delivery. Nucleic Acids Res. (2017) 45:e98. doi: 10.1093/nar/gkx154

54. Eyquem J, Mansilla-Soto J, Giavridis T, van der Stegen SJC, Hamieh $\mathrm{M}$, Cunanan KM, et al. Targeting a CAR to the TRAC locus with CRISPR/Cas9 enhances tumour rejection. Nature. (2017) 543:113-7. doi: 10.1038 /nature21405

55. Pulè MA, Straathof KC, Dotti G, Heslop HE, Rooney CM, Brenner MK. A chimeric $\mathrm{T}$ cell antigen receptor that augments cytokine release and supports clonal expansion of primary human T cells. Mol Ther J Am Soc Gene Ther. (2005) 12:933-41. doi: 10.1016/j.ymthe.2005.04.016

56. Grupp SA, Mitchell RN, Schreiber KL, McKean DJ, Abbas AK. Molecular mechanisms that control expression of the B lymphocyte antigen receptor complex. J Exp Med. (1995) 181:161-8. doi: 10.1084/jem.181.1.161

57. Sanchez M, Misulovin Z, Burkhardt AL, Mahajan S, Costa T, Franke R, et al. Signal transduction by immunoglobulin is mediated through Ig alpha and Ig beta. J Exp Med. (1993) 178:1049-55. doi: 10.1084/jem.178.3.1049

58. Sather BD, Romano Ibarra GS, Sommer K, Curinga G, Hale M, Khan IF, et al. Efficient modification of CCR5 in primary human hematopoietic cells using a megaTAL nuclease and AAV donor template. Sci Transl Med. (2015) 7:307ra156. doi: 10.1126/scitranslmed.aac5530

59. Roth TL, Puig-Saus C, Yu R, Shifrut E, Carnevale J, Li PJ, et al. Reprogramming human $\mathrm{T}$ cell function and specificity with non-viral genome targeting. Nature. (2018) 559:405-9. doi: 10.1038/s41586-018-0326-5

60. Kelton W, Waindok AC, Pesch T, Pogson M, Ford K, Parola C, et al. Reprogramming MHC specificity by CRISPR-Cas9-assisted cassette exchange. Sci Rep. (2017) 7:45775. doi: 10.1038/srep45775

61. Barzel A, Paulk NK, Shi Y, Huang Y, Chu K, Zhang F, et al. Promoterless gene targeting without nucleases ameliorates haemophilia B in mice. Nature. (2015) 517:360-4. doi: 10.1038/nature13864

62. Mierau M, Drexler GA, Kutzera A, Braunschmidt K, Ellwart J, EckardtSchupp F, et al. Non-conservative homologous recombination in human B lymphocytes is promoted by activation-induced cytidine deaminase and transcription. Nucleic Acids Res. (2008) 36:5591-601. doi: 10.1093/nar/gkn542

63. Hartweger H, McGuire AT, Horning M, Taylor JJ, Dosenovic P, Yost D, et al. HIV-specific humoral immune responses by CRISPR/Cas9-edited B cells. $J$ Exp Med. (2019) 216:1301-10. doi: 10.1084/jem.20190287

64. Voss JE, Gonzalez-Martin A, Andrabi R, Fuller RP, Murrell B, McCoy LE, et al. Reprogramming the antigen specificity of B cells using genome-editing technologies. eLife. (2019) 8:e42995. doi: 10.7554/eLife.42995

65. Moffett HF, Harms CK, Fitzpatrick KS, Tooley MR, Boonyaratanakornkit J, Taylor JJ. B cells engineered to express pathogen-specific antibodies protect against infection. Sci Immunol. (2019) 4:eaax0644. doi: 10.1126/sciimmunol.aax0644

66. Pesch T, Bonati L, Kelton W, Parola C, Ehling RA, Csepregi L, et al. Molecular design, optimization and genomic integration of chimeric $\mathrm{B}$ cell receptors in murine B cells. bioRxvi (2019). doi: 10.1101/516369

Conflict of Interest: The authors declare that the research was conducted in the absence of any commercial or financial relationships that could be construed as a potential conflict of interest.

Copyright (๔ 2019 Pesch, Bonati, Kelton, Parola, Ehling, Csepregi, Kitamura and Reddy. This is an open-access article distributed under the terms of the Creative Commons Attribution License (CC BY). The use, distribution or reproduction in other forums is permitted, provided the original author(s) and the copyright owner(s) are credited and that the original publication in this journal is cited, in accordance with accepted academic practice. No use, distribution or reproduction is permitted which does not comply with these terms. 